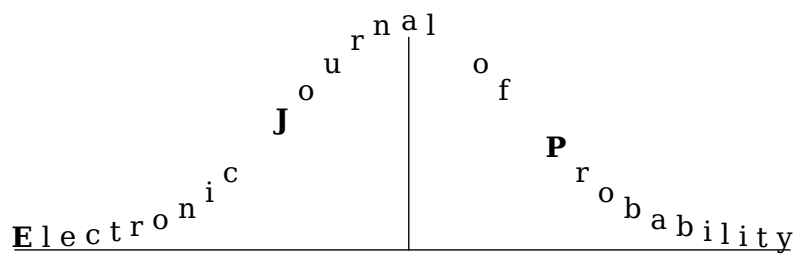

Electron. J. Probab. 26 (2021), article no. 58, 1-21.

ISSN: 1083-6489 https://doi.org/10.1214/21-EJP618

\title{
The area under a spectrally positive stable excursion and other related processes
}

\author{
Christophe Profeta*
}

\begin{abstract}
We study the distribution of the area under the normalized excursion of a spectrally positive stable Lévy process $L$, as well as the area under its meander, and under $L$ conditioned to stay positive. Our results involve a special case of Wright's function, which may be seen as a generalization of the classic Airy function appearing in similar Brownian's areas.
\end{abstract}

Keywords: stable processes; normalized excursion; meander MSC2020 subject classifications: 60G52; 60G18; 60E10.

Submitted to EJP on September 5, 2020, final version accepted on March 29, 2021.

\section{Introduction}

Let $L$ be an $\alpha$-stable Lévy process without negative jumps, with $\alpha>1$. We assume that $L$ is normalized to have characteristic exponent:

$$
\ln \left(\mathbb{E}\left[e^{i \lambda L_{1}}\right]\right)=(i \lambda)^{\alpha} e^{-i \pi \alpha \operatorname{sgn}(\lambda)}, \quad \lambda \in \mathbb{R} .
$$

It is well-known, see for instance [22, Prop. 3.4.1], that the distribution of the area under $L$ also follows a stable distribution:

$$
\int_{0}^{1} L_{u} d u \stackrel{\text { (law) }}{=}(1+\alpha)^{-\frac{1}{\alpha}} L_{1} .
$$

The purpose of this note is to study the distribution of the area under three related processes: the normalized excursion of $L$, the meander of $L$, and $L$ conditioned to stay positive. These distributions have already been extensively studied in the Brownian case, see in particular the survey by Janson [8] or the paper by Perman \& Wellner [21]. For all these Brownian areas, one observes the occurrence of the classic Airy function Ai. We

*Université Paris-Saclay, CNRS, Univ Evry, Laboratoire de Mathématiques et Modélisation d'Evry, 91037, Evry-Courcouronnes, France. E-mail: christophe.profeta@univ-evry.fr 
shall prove that for spectrally positive stable Lévy processes, the role of Ai is played by the following M-Wright's function

$$
\Phi_{\alpha}(x)=\frac{1}{\pi} \sum_{n=0}^{+\infty} \frac{(-1)^{n}}{n !} \Gamma\left(\frac{1+n}{1+\alpha}\right) \sin \left(\pi \frac{1+n}{\alpha+1}\right)(1+\alpha)^{\frac{n-\alpha}{1+\alpha}} x^{n} .
$$

The function $\Phi_{\alpha}$ is known to be related to a time-fractional diffusion equation, see [18] and the references within. In particular, it admits the integral representation:

$$
\Phi_{\alpha}(x)=\frac{1}{\pi} \int_{0}^{+\infty} e^{-\sin \left(\frac{\pi \alpha}{2}\right) \frac{z^{1+\alpha}}{1+\alpha}} \cos \left(\cos \left(\frac{\pi \alpha}{2}\right) \frac{z^{1+\alpha}}{1+\alpha}-z x\right) d z
$$

from which we immediately see that $\Phi_{2}=\mathrm{Ai}$. Another function of interest will be its cosine counterpart:

$$
\Psi_{\alpha}(x)=\frac{1}{\pi} \sum_{n=0}^{+\infty} \frac{(-1)^{n}}{n !} \Gamma\left(\frac{1+n}{1+\alpha}\right) \cos \left(\pi \frac{1+n}{\alpha+1}\right)(1+\alpha)^{\frac{n-\alpha}{1+\alpha}} x^{n}
$$

We now state the main results of this paper.

\subsection{The area under a normalized excursion}

Let $L^{(\mathrm{ex})}$ denote a normalized excursion of $L$ on the segment $[0,1]$ and set

$$
\mathcal{A}_{\mathrm{ex}}=\int_{0}^{1} L_{t}^{(\mathrm{ex})} d t
$$

Theorem 1.1. The double Laplace transform of $\mathcal{A}_{\mathrm{ex}}$ is given by:

$$
\int_{0}^{+\infty}\left(1-e^{-\lambda t}\right) \mathbb{E}\left[e^{-t^{1+\frac{1}{\alpha}} \mathcal{A}_{\mathrm{ex}}}\right] t^{-\left(1+\frac{1}{\alpha}\right)} d t=\alpha \Gamma\left(1-\frac{1}{\alpha}\right)\left(\frac{\Phi_{\alpha}^{\prime}(0)}{\Phi_{\alpha}(0)}-\frac{\Phi_{\alpha}^{\prime}(\lambda)}{\Phi_{\alpha}(\lambda)}\right) .
$$

In the Brownian case, i.e. when $\alpha=2$, the distribution of $\mathcal{A}_{\text {ex }}$ is nowadays designated in the literature as the Airy distribution. We refer the reader to Louchard [16, 17] for a study of the Brownian excursion area via the Feynman-Kac formula, to Takács [24] for an approach via random walks and to Flajolet \& Louchard [6] for a study of Airy distribution via its moments.

In the physics literature, this distribution has also appeared in the study of fluctuating interfaces, see [19]. We finally mention that more recently, some authors have investigated the area under a normalized Bessel excursion and shown its relation with the cooling of atoms $[1,12]$.

As is the case for the Airy distribution, we may deduce from Theorem 1.1 a recurrence relation for the moments of $\mathcal{A}_{\text {ex }}$. To this end, let us define a sequence $\left(B_{n, k}, 1 \leq n, 1 \leq\right.$ $k \leq n) \mathrm{by}^{1}$

$$
B_{n, 1}=\frac{(2-\alpha)_{n-1}}{(n+1)(n+2)}, \quad n \geq 1,
$$

and for $k \geq 1$,

$$
B_{n, k+1}=\frac{1}{k+1} \sum_{l=k}^{n-1}\left(\begin{array}{l}
n \\
l
\end{array}\right) B_{n-l, 1} \times B_{l, k}, \quad n \geq 1 .
$$

\footnotetext{
${ }^{1}$ For $x \in \mathbb{R}$ and $n \in \mathbb{N},(x)_{n}=x(x+1) \ldots(x+n-1)$ denotes the usual Pochammer symbol, with the convention $(x)_{0}=1$.
} 
This sequence corresponds to the values of the exponential partial Bell polynomials taken on the sequence $\left(B_{n, 1}, n \geq 1\right)$, see Comtet [5, Section 3.3]. We then set $c_{0}^{(\alpha)}=1$ and for $p \geq 1$,

$$
c_{p}^{(\alpha)}=\frac{1}{(2 p) ! \sqrt{\pi}}\left(\frac{2}{\alpha}\right)^{p} \sum_{k=1}^{2 p} B_{2 p, k} \Gamma\left(p+k+\frac{1}{2}\right)(2(\alpha-1))^{k} .
$$

The coefficients $\left(c_{p}^{(\alpha)}, p \geq 0\right)$ are the ones appearing in the asymptotic expansion of the function $\Phi_{\alpha}$, see Proposition A.2 in the Appendix.

Corollary 1.2. The moments of the random variable $\mathcal{A}_{\mathrm{ex}}$ are given by:

$$
\mathbb{E}\left[\mathcal{A}_{\mathrm{ex}}^{n}\right]=n ! \Omega_{n} \alpha \Gamma\left(1-\frac{1}{\alpha}\right) / \Gamma\left(\frac{(n-1)(\alpha+1)}{\alpha}+1\right)
$$

where the sequence $\left(\Omega_{n}, n \geq 1\right)$ is defined recursively by $\Omega_{1}=\frac{\alpha-1}{2 \alpha}$ and for $n \geq 2$,

$$
\Omega_{n}=c_{n-1}^{(\alpha)} \frac{(2 n-1)(\alpha+1)-2}{2 \alpha}-\sum_{k=1}^{n-1} \Omega_{k} c_{n-k}^{(\alpha)} .
$$

As a consequence, we have the asymptotics ${ }^{2}$ :

$$
\left(\mathbb{E}\left[\mathcal{A}_{\mathrm{ex}}^{n}\right]\right)^{\frac{1}{n}} \underset{n \rightarrow+\infty}{\asymp} n^{1-\frac{1}{\alpha}} \quad \text { and } \quad \ln \mathbb{P}\left(\mathcal{A}_{\mathrm{ex}}>x\right) \underset{x \rightarrow+\infty}{\asymp}-x^{\frac{\alpha}{\alpha-1}} .
$$

The first moments are given by:

$$
\mathbb{E}\left[\mathcal{A}_{\mathrm{ex}}\right]=\frac{\alpha-1}{2} \Gamma\left(1-\frac{1}{\alpha}\right) \quad \text { and } \quad \mathbb{E}\left[\mathcal{A}_{\mathrm{ex}}^{2}\right]=\frac{\Gamma\left(1-\frac{1}{\alpha}\right)(\alpha-1)(2 \alpha+1)}{12 \Gamma\left(1+\frac{1}{\alpha}\right)} .
$$

Note that in the Brownian case, since $B_{n, 1}=0$ as soon as $n \geq 2$, the coefficients $c_{n}^{(2)}$ simplify to

$$
c_{n}^{(2)}=\frac{1}{(2 n) ! \sqrt{\pi}}\left(\frac{1}{3}\right)^{2 n} \Gamma\left(3 n+\frac{1}{2}\right)
$$

and we recover a classic recurrence formula for the moments of Airy distribution, see [17]. In this case, a complete asymptotic expansion for $\mathbb{P}\left(\mathcal{A}_{\mathrm{ex}}>x\right)$ was computed by Janson \& Louchard in [10].

\subsection{The area under a stable meander}

Let $L^{(\mathrm{me})}$ denote the meander of $L$ on the segment $[0,1]$ and set

$$
\mathcal{A}_{\mathrm{me}}=\int_{0}^{1} L_{t}^{(\mathrm{me})} d t
$$

Theorem 1.3. The double Laplace transform of $\mathcal{A}_{\mathrm{me}}$ is given by:

$$
\int_{0}^{+\infty} e^{-\lambda t} \mathbb{E}\left[e^{-t^{1+\frac{1}{\alpha}} \mathcal{A}_{\mathrm{me}}}\right] t^{-\frac{1}{\alpha}} d t=\pi \Gamma\left(1-\frac{1}{\alpha}\right) \frac{\Psi_{\alpha}^{\prime}(\lambda) \Phi_{\alpha}(\lambda)-\Phi_{\alpha}^{\prime}(\lambda) \Psi_{\alpha}(\lambda)}{\Phi_{\alpha}(\lambda)} .
$$

In particular,

$$
\mathbb{E}\left[\mathcal{A}_{\mathrm{me}}\right]=\Gamma\left(1-\frac{1}{\alpha}\right) \frac{\alpha+1}{2 \alpha}
$$

and there is the asymptotics, for $1<\alpha<2$ :

$$
\mathbb{P}\left(\mathcal{A}_{\mathrm{me}}>x\right) \underset{x \rightarrow+\infty}{\sim} \frac{(\alpha-1) \Gamma(1+\alpha) \Gamma\left(1-\frac{1}{\alpha}\right)}{\Gamma(2-\alpha) \Gamma\left(2+\alpha-\frac{1}{\alpha}\right)} x^{-\alpha} .
$$

\footnotetext{
${ }^{2}$ We write $a_{p} \asymp b_{p}$ as $p \rightarrow+\infty$ to state that there exist two constants $0<\kappa_{1} \leq \kappa_{2}<+\infty$ such that $\kappa_{1} a_{p} \leq b_{p} \leq \kappa_{2} a_{p}$ for $p$ large enough.
} 
In the Brownian case, this distribution was studied for instance by Takács [25], see also Perman \& Wellner [21]. Note that here, since the process is not pinned down at $t=1$, the presence of positive jumps yields a polynomial decay of the tail of $\mathcal{A}_{\text {me }}$, which is different from the case $\alpha=2$, see Janson $\&$ Louchard [10].

Remark 1.4. Again, when $\alpha=2$, Theorem 1.3 simplifies. Indeed, observe that in this case, $\Psi_{2}$ is a solution of the following differential equation

$$
\Psi_{2}^{\prime \prime}(\lambda)=\lambda \Psi_{2}(\lambda)-\frac{1}{\pi}
$$

hence the derivative of the numerator on the right-hand side of (1.5) equals

$$
\left(\Psi_{2}^{\prime}(\lambda) \operatorname{Ai}(\lambda)-\operatorname{Ai}^{\prime}(\lambda) \Psi_{2}(\lambda)\right)^{\prime}=-\frac{1}{\pi} \operatorname{Ai}(\lambda) .
$$

By integration, this implies that

$$
\Psi_{2}^{\prime}(\lambda) \operatorname{Ai}(\lambda)-\operatorname{Ai}^{\prime}(\lambda) \Psi_{2}(\lambda)=\frac{1}{3 \pi}-\frac{1}{\pi} \int_{0}^{\lambda} \operatorname{Ai}(x) d x=\frac{1}{\pi} \int_{\lambda}^{+\infty} \operatorname{Ai}(x) d x
$$

which agrees for instance with [21, Corollary 3.2].

\subsection{The area under $L$ conditioned to stay positive}

Let $L^{\uparrow}$ be the process $L$ started from 0 and conditioned to stay positive. We set

$$
\mathcal{A}^{\uparrow}=\int_{0}^{1} L_{t}^{\uparrow} d t
$$

Theorem 1.5. The double Laplace transform of $\mathcal{A}^{\uparrow}$ is given by:

$$
\int_{0}^{+\infty} e^{-\lambda t} \mathbb{E}\left[e^{-t^{1+\frac{1}{\alpha}} \mathcal{A}^{\uparrow}}\right] d t=\pi \lambda \frac{\Phi_{\alpha}^{\prime}(\lambda) \Psi_{\alpha}(\lambda)-\Psi_{\alpha}^{\prime}(\lambda) \Phi_{\alpha}(\lambda)}{\Phi_{\alpha}(\lambda)}-\frac{\Phi_{\alpha}^{\prime}(\lambda)}{\Phi_{\alpha}(\lambda)} .
$$

In particular, there is the asymptotics, for $1<\alpha<2$ :

$$
\mathbb{P}\left(\mathcal{A}^{\uparrow}>x\right) \underset{x \rightarrow+\infty}{\sim} \frac{\Gamma(1+\alpha)}{\Gamma\left(1+\alpha-\frac{1}{\alpha}\right) \Gamma(2-\alpha)} x^{1-\alpha} .
$$

In the Brownian case, $\mathcal{A}^{\uparrow}$ corresponds, up to a $\sqrt{2}$ factor, to the integral on $[0,1]$ of a three-dimensional Bessel process started from 0. As for the meander, Formula (1.6) simplifies when $\alpha=2$, and the right-hand side equals

$$
-\lambda \frac{\int_{\lambda}^{+\infty} \operatorname{Ai}(x) d x}{\operatorname{Ai}(\lambda)}-\frac{\operatorname{Ai}^{\prime}(\lambda)}{\operatorname{Ai}(\lambda)}=\frac{-\lambda \int_{\lambda}^{+\infty} \operatorname{Ai}(x) d x+\int_{\lambda}^{+\infty} x \operatorname{Ai}(x) d x}{\operatorname{Ai}(\lambda)}=\frac{\int_{0}^{+\infty} x \operatorname{Ai}(x+\lambda) d x}{\operatorname{Ai}(\lambda)}
$$

which agrees with [3, p. 440].

\subsection{Outline of the paper}

The remainder of the paper is divided as follows. Section 2 provides some notation as well as a key proposition which is of independent interest. Sections 3, 4 and 5 give the proofs of the main Theorems, respectively on the normalized excursion, the meander, and $L$ conditioned to stay positive. Finally, Appendix A is an appendix on the Wright's function $\Phi_{\alpha}$, where we compute its asymptotic expansion at infinity. 


\section{Preliminaries}

\subsection{Notations}

We start by recalling the definition of the considered processes, for which we mainly refer to Chaumont [4]. We assume that $L$ is defined on the Skorokhod space of càdlàg processes. We denote by $\mathbb{P}_{x}$ its law when $L_{0}=x$, with the convention that $\mathbb{P}=\mathbb{P}_{0}$, and by $\left(\mathcal{F}_{t}, t \geq 0\right)$ its natural filtration. Define, for $z \in \mathbb{R}$,

$$
T_{z}=\inf \left\{t \geq 0, L_{t}=z\right\} \text {. }
$$

1. We denote by $\mathbb{P}_{x, y}^{t}$ the law of the bridge of $L$ of length $t$, going from $x$ to $y$.

2. We denote by $\mathbb{P}_{x}^{\uparrow}$ the law of $L$ started at $x>0$ and conditioned to stay positive. It is classically given by the $h$-transform:

$$
\forall \Lambda_{t} \in \mathcal{F}_{t}, \quad \mathbb{P}_{x}^{\uparrow}\left(\Lambda_{t}\right)=\frac{1}{x} \mathbb{E}_{x}\left[L_{t} 1_{\Lambda_{t}} 1_{\left\{T_{0}>t\right\}}\right] .
$$

This law admits a weak limit in the Skorokhod sense as $x \downarrow 0$ which we shall denote $\mathbb{P}_{0}^{\uparrow}$.

3. We denote by $\mathbb{P}^{(m e)}$ the law of the meander of $L$, which is given by the limit

$$
\forall \Lambda_{t} \in \mathcal{F}_{t}, \quad \mathbb{P}^{(m e)}\left(\Lambda_{t}\right)=\lim _{x \downarrow 0} \mathbb{P}_{x}\left(\Lambda_{t} \mid T_{0}>t\right)
$$

4. Finally, we recall that, since $L$ is spectrally positive, the law of the stable excursion of length $t$ is equivalent to the bridge of $L$ conditioned to stay positive, and starting and ending at 0 . From Lemma 4 in [4], this law may be for instance defined by

$$
\forall s<t \quad \Lambda_{s} \in \mathcal{F}_{s}, \quad \mathbb{P}_{0,0}^{\uparrow t}\left(\Lambda_{s}\right)=\lim _{x \downarrow 0} \mathbb{P}_{x}\left(\Lambda_{s} \mid T_{0}=t\right)
$$

\subsection{The key proposition}

The proofs of Theorems 1.1, 1.3 and 1.5 will rely heavily on the following proposition, which gives the joint Laplace transform of the pair $\left(T_{0}, \int_{0}^{T_{0}} L_{s} d s\right)$.

Proposition 2.1. For $z, \lambda \geq 0$ and $\mu>0$ we have:

$$
\mathbb{E}_{z}\left[e^{-\lambda T_{0}-\mu \int_{0}^{T_{0}} L_{s} d s}\right]=\frac{\Phi_{\alpha}\left(\mu^{\frac{1}{1+\alpha}}\left(z+\frac{\lambda}{\mu}\right)\right)}{\Phi_{\alpha}\left(\mu^{-\frac{\alpha}{1+\alpha}} \lambda\right)} .
$$

Proof. We first assume that $\lambda=0$. The law of $\int_{0}^{T_{0}} L_{s} d s$ has been studied by Letemplier $\&$ Simon in [15]. In particular, they obtain the Mellin transform:

$$
\mathbb{E}_{1}\left[\left(\int_{0}^{T_{0}} L_{s} d s\right)^{\nu}\right]=(\alpha+1)^{\nu} \frac{\Gamma\left(\frac{\alpha}{\alpha+1}\right) \Gamma(1-(\alpha+1) \nu)}{\Gamma\left(\frac{\alpha}{\alpha+1}-\nu\right) \Gamma(1-\nu)}, \quad \nu<\frac{1}{1+\alpha} .
$$

Replacing $\nu$ by $-\nu$ and using the definition of the Gamma function, we obtain:

$$
\int_{0}^{+\infty} \mu^{\nu-1} \mathbb{E}_{1}\left[e^{-\mu \int_{0}^{T_{0}} L_{s} d s}\right] d \mu=(\alpha+1)^{-\nu} \frac{\Gamma(\nu) \Gamma\left(\frac{\alpha}{\alpha+1}\right) \Gamma(1+(\alpha+1) \nu)}{\Gamma\left(\frac{\alpha}{\alpha+1}+\nu\right) \Gamma(1+\nu)} .
$$

We now invert this Mellin transform following Janson [9]:

$$
\mathbb{E}_{1}\left[e^{-\mu \int_{0}^{T_{0}} L_{s} d s}\right]=1+\Gamma\left(\frac{\alpha}{1+\alpha}\right) \sum_{n=1}^{+\infty} \frac{(-1)^{n}}{n !} \frac{(1+\alpha)^{\frac{n}{1+\alpha}}}{\Gamma\left(\frac{\alpha-n}{\alpha+1}\right)} \mu^{\frac{n}{1+\alpha}}
$$


hence, by scaling, we thus obtain

$$
\mathbb{E}_{z}\left[e^{-\mu \int_{0}^{T_{0}} L_{s} d s}\right]=\Gamma\left(\frac{\alpha}{1+\alpha}\right)(1+\alpha)^{\frac{\alpha}{1+\alpha}} \Phi_{\alpha}\left(z \mu^{\frac{1}{1+\alpha}}\right)=\frac{\Phi_{\alpha}\left(z \mu^{\frac{1}{1+\alpha}}\right)}{\Phi_{\alpha}(0)}
$$

which is Proposition 2.1 when $\lambda=0$. We now deal with the general case. Let $x>y>0$. Applying the Markov property, we deduce from the absence of negative jumps that

$$
\mathbb{E}_{x}\left[e^{-\mu \int_{0}^{T_{0}} L_{s} d s}\right]=\mathbb{E}_{x}\left[e^{-\mu \int_{0}^{T_{y}} L_{s} d s}\right] \times \mathbb{E}_{y}\left[e^{-\mu \int_{0}^{T_{0}} L_{s} d s}\right] .
$$

Furthermore, by translation, we also have:

$$
\mathbb{E}_{x}\left[e^{-\mu \int_{0}^{T_{y}} L_{s} d s}\right]=\mathbb{E}_{x-y}\left[e^{-\mu y T_{0}-\mu \int_{0}^{T_{0}} L_{s} d s}\right] .
$$

Finally, setting $x-y=z>0$ and $\lambda=\mu y>0$, and plugging (2.3) in (2.4) yields

$$
\mathbb{E}_{z}\left[e^{-\lambda T_{0}-\mu \int_{0}^{T_{0}} L_{s} d s}\right]=\frac{\mathbb{E}_{x}\left[e^{-\mu \int_{0}^{T_{0}} L_{s} d s}\right]}{\mathbb{E}_{y}\left[e^{-\mu \int_{0}^{T_{0}} L_{s} d s}\right]}=\frac{\Phi_{\alpha}\left(\mu^{\frac{1}{1+\alpha}}\left(z+\frac{\lambda}{\mu}\right)\right)}{\Phi_{\alpha}\left(\mu^{-\frac{\alpha}{1+\alpha}} \lambda\right)}
$$

which ends the proof of Proposition 2.1.

Remark 2.2. Proposition 2.1 was proven in the Brownian case by Lefebvre [14], by applying the Feynman-Kac formula. A generalization where $T_{0}$ is replaced by the exit time from an interval was obtained by Lachal [13] by a similar method.

\section{The area under a spectrally positive stable excursion}

\subsection{Proof of Theorem 1.1: the double Laplace transform}

Notice first that by monotone convergence, the absolute continuity formula (2.2) remains true at $s=t$ :

$$
\mathbb{E}_{0,0}^{\uparrow t}\left[e^{-\int_{0}^{t} L_{u} d u}\right]=\lim _{x \downarrow 0} \mathbb{E}_{x}\left[e^{-\int_{0}^{t} L_{u} d u} \mid T_{0}=t\right] .
$$

Next, starting from Proposition 2.1, we may write

$$
\frac{\Phi_{\alpha}(z)}{\Phi_{\alpha}(0)}-\frac{\Phi_{\alpha}(z+\lambda)}{\Phi_{\alpha}(\lambda)}=\int_{0}^{+\infty}\left(1-e^{-\lambda t}\right) \mathbb{E}_{z}\left[e^{-\int_{0}^{t} L_{u} d u} \mid T_{0}=t\right] \mathbb{P}_{z}\left(T_{0} \in d t\right) .
$$

Recall now from Sato [23, Theorem 46.3] that since $L$ has no negative jumps, $T_{0}$ is a positive stable random variable of index $1 / \alpha$, i.e. for $z>0$ :

$$
\mathbb{P}_{z}\left(T_{0} \in d t\right) / d t=\frac{1}{\pi} \sum_{n=1}^{+\infty} \frac{(-1)^{n-1}}{n !} \sin (n \pi / \alpha) \Gamma(1+n / \alpha) z^{n} t^{-1-n / \alpha} .
$$

Dividing (3.2) by $z$ and letting $z \downarrow 0$, we deduce from (3.1) and (3.3) that

$$
\frac{\Phi_{\alpha}^{\prime}(0)}{\Phi_{\alpha}(0)}-\frac{\Phi_{\alpha}^{\prime}(\lambda)}{\Phi_{\alpha}(\lambda)}=\frac{\sin (\pi / \alpha)}{\pi} \Gamma\left(1+\frac{1}{\alpha}\right) \int_{0}^{+\infty}\left(1-e^{-\lambda t}\right) \mathbb{E}_{0,0}^{\uparrow t}\left[e^{-\int_{0}^{t} L_{u} d u}\right] t^{-1-\frac{1}{\alpha}} d t .
$$

Theorem 1.1 now follows by the complement formula for the Gamma function and the scaling property. 


\subsection{Proof of Corollary 1.2: study of the positive moments}

To get information on the moments, we shall work with a slight modification of the formula of Theorem 1.1. Indeed, differentiating under the integral sign, we deduce that for $\lambda>0$ :

$$
\int_{0}^{+\infty} e^{-\lambda t} \mathbb{E}\left[e^{-t^{1+\frac{1}{\alpha}}} \mathcal{A}_{\mathrm{ex}}\right] \frac{d t}{t^{\frac{1}{\alpha}}}=\Gamma\left(-\frac{1}{\alpha}\right)\left(\frac{\Phi_{\alpha}^{\prime \prime}(\lambda)}{\Phi_{\alpha}(\lambda)}-\left(\frac{\Phi_{\alpha}^{\prime}(\lambda)}{\Phi_{\alpha}(\lambda)}\right)^{2}\right) .
$$

As a consequence, we obtain:

$$
\int_{0}^{+\infty} e^{-\lambda t} \mathbb{E}\left[1-e^{-t^{1+\frac{1}{\alpha}}} \mathcal{A}_{\mathrm{ex}}\right] \frac{d t}{t^{\frac{1}{\alpha}}}=\frac{\Gamma\left(1-\frac{1}{\alpha}\right)}{\lambda^{1-\frac{1}{\alpha}}}-\Gamma\left(-\frac{1}{\alpha}\right)\left(\frac{\Phi_{\alpha}^{\prime \prime}(\lambda)}{\Phi_{\alpha}(\lambda)}-\left(\frac{\Phi_{\alpha}^{\prime}(\lambda)}{\Phi_{\alpha}(\lambda)}\right)^{2}\right) .
$$

We now integrate this relation with respect to $\lambda$ on $(\xi,+\infty)$. Using the Fubini-Tonelli theorem on the left-hand side, and the asymptotics of $\Phi_{\alpha}$ and $\Phi_{\alpha}^{\prime}$ given by Proposition A.2 in the Appendix on the right-hand side, we deduce that

$$
\int_{0}^{+\infty} e^{-\xi t} \mathbb{E}\left[1-e^{-t^{1+\frac{1}{\alpha}}} \mathcal{A}_{\mathrm{ex}}\right] \frac{d t}{t^{1+\frac{1}{\alpha}}}=\Gamma\left(-\frac{1}{\alpha}\right) \xi^{1 / \alpha}+\Gamma\left(-\frac{1}{\alpha}\right) \frac{\Phi_{\alpha}^{\prime}(\xi)}{\Phi_{\alpha}(\xi)} .
$$

Setting $x=\xi^{-(1+1 / \alpha)}$ and making the change of variable $s=\lambda t$ then yields

$$
\int_{0}^{+\infty} e^{-s} \mathbb{E}\left[1-e^{-x s^{1+\frac{1}{\alpha}}} \mathcal{A}_{\mathrm{ex}}\right] \frac{d s}{s^{1+\frac{1}{\alpha}}}=\Gamma\left(-\frac{1}{\alpha}\right)\left(x^{\frac{1}{1+\alpha}} \frac{\Phi_{\alpha}^{\prime}\left(x^{-\frac{\alpha}{1+\alpha}}\right)}{\Phi_{\alpha}\left(x^{-\frac{\alpha}{1+\alpha}}\right)}+1\right) .
$$

Next, observe from Proposition A.2 that the right-hand side admits the asymptotic expansion, as $x \downarrow 0$ :

$$
\begin{aligned}
\Gamma\left(-\frac{1}{\alpha}\right)\left(x^{\frac{1}{1+\alpha}} \frac{\Phi_{\alpha}^{\prime}\left(x^{-\frac{\alpha}{1+\alpha}}\right)}{\Phi_{\alpha}\left(x^{-\frac{\alpha}{1+\alpha}}\right)}+1\right) & =\frac{\sum_{p=0}^{n}(-1)^{p+1} d_{p}^{(\alpha)} x^{p}+\mathrm{o}\left(x^{n}\right)}{\sum_{p=0}^{n}(-1)^{p} c_{p}^{(\alpha)} x^{p}+\mathrm{o}\left(x^{n}\right)}+1 \\
& =\Gamma\left(-\frac{1}{\alpha}\right) \sum_{k=1}^{n}(-1)^{n} \Omega_{k} x^{k}+\mathrm{o}\left(x^{n+1}\right)
\end{aligned}
$$

where, using a Cauchy product, the sequence $\left(\Omega_{k}\right)$ is given by the recurrence relation (1.4). We shall prove by induction that for any $k \geq 1, \mathbb{E}\left[\mathcal{A}_{\mathrm{ex}}^{k}\right]$ is finite and equals

$$
\mathbb{E}\left[\mathcal{A}_{\mathrm{ex}}^{k}\right]=k ! \Omega_{k} \alpha \Gamma\left(1-\frac{1}{\alpha}\right) / \Gamma\left(\frac{(k-1)(\alpha+1)}{\alpha}+1\right) .
$$

For $k=1$, we obtain applying the Fubini-Tonelli theorem in Equation (3.4):

$$
\int_{0}^{+\infty} e^{-s} \int_{0}^{+\infty} e^{-x s^{1+\frac{1}{\alpha}} z} \mathbb{P}\left(\mathcal{A}_{\mathrm{ex}} \geq z\right) d z d s=\alpha \Gamma\left(1-\frac{1}{\alpha}\right) \Omega_{1}+\mathrm{o}(1) .
$$

Then, letting $x \downarrow 0$ and applying the monotone convergence theorem on the left-hand side, we deduce that the expectation of $\mathcal{A}_{\mathrm{ex}}$ is finite and equals:

$$
\mathbb{E}\left[\mathcal{A}_{\mathrm{ex}}\right]=\alpha \Gamma\left(1-\frac{1}{\alpha}\right) \Omega_{1}=\frac{\alpha-1}{2} \Gamma\left(1-\frac{1}{\alpha}\right) .
$$

Next, let $n \in \mathbb{N}$ and assume now that Formula (3.5) holds true for all $k \leq n$. Plugging the Taylor formula with integral remainder of the exponential function

$$
\begin{aligned}
1 & -e^{-x s^{1+\frac{1}{\alpha}} \mathcal{A}_{\mathrm{ex}}} \\
& =\sum_{k=1}^{n}(-1)^{k-1} \frac{x^{k}}{k !}\left(s^{1+\frac{1}{\alpha}} \mathcal{A}_{\mathrm{ex}}\right)^{k}+(-1)^{n} \frac{x^{n+1}}{n !}\left(s^{1+\frac{1}{\alpha}} \mathcal{A}_{\mathrm{ex}}\right)^{n+1} \int_{0}^{1}(1-t)^{n} e^{-t s^{1+\frac{1}{\alpha}} x \mathcal{A}_{\mathrm{ex}}} d t
\end{aligned}
$$


The area under a spectrally positive stable excursion

into Equation (3.4), and using the recurrence assumption, we deduce that:

$$
\frac{1}{n !} \int_{0}^{+\infty} e^{-s} s^{n\left(1+\frac{1}{\alpha}\right)} \int_{0}^{1}(1-t)^{n} \mathbb{E}\left[\mathcal{A}_{\mathrm{ex}}^{n+1} e^{-t s^{1+\frac{1}{\alpha}} x \mathcal{A}_{\mathrm{ex}}}\right] d t d s=\alpha \Gamma\left(1-\frac{1}{\alpha}\right) \Omega_{n+1}+\mathrm{o}(1) .
$$

Then, letting $x \downarrow 0$ and applying once again the monotone convergence theorem on the left-hand side, we conclude that

$$
\frac{1}{(n+1) !} \Gamma\left(\frac{n(\alpha+1)}{\alpha}+1\right) \mathbb{E}\left[\mathcal{A}_{\mathrm{ex}}^{n+1}\right]=\alpha \Gamma\left(1-\frac{1}{\alpha}\right) \Omega_{n+1}
$$

which is Formula (3.5) with $k=n+1$.

\subsection{Proof of Corollary 1.2: asymptotics}

To study the asymptotics of $\left(\Omega_{n}\right)$, observe first that by definition, this sequence is positive, and so is the sequence $\left(c_{n}^{(\alpha)}\right)$. Therefore, we deduce from the recurrence relation (1.4) and from Corollary A.3 in the Appendix that there exists a finite constant $\kappa_{1}$ such that for $n$ large enough

$$
\Omega_{n}^{\frac{1}{n}} \leq\left(c_{n-1}^{(\alpha)} \frac{(2 n-1)(\alpha+1)-2}{2 \alpha}\right)^{\frac{1}{n}} \leq \kappa_{1} n .
$$

Stirling's formula now implies the upper bound for $\mathbb{E}\left[\mathcal{A}_{\mathrm{ex}}^{n}\right]^{\frac{1}{n}}$, which may be written

$$
\limsup _{n \rightarrow+\infty} \frac{\mathbb{E}\left[\mathcal{A}_{\mathrm{ex}}^{n}\right]^{\frac{1}{n}}}{n^{1-\frac{1}{\alpha}}} \leq \kappa_{2}
$$

for some finite constant $\kappa_{2}>0$. To obtain the tail decay of $\mathcal{A}_{\text {ex }}$, we shall rely on Kasahara's Tauberian theorem of exponential type. Indeed, applying [11, Theorem 4], we deduce from (3.6) that there exists a constant $0<\kappa_{3}<+\infty$ such that

$$
\limsup _{x \rightarrow+\infty} \frac{1}{x} \ln \mathbb{P}\left(\mathcal{A}_{\mathrm{ex}}>x^{1-\frac{1}{\alpha}}\right) \leq-\kappa_{3}
$$

which gives the announced upper bound.

It does not seem easy to obtain a lower bound for $\Omega_{n}$ from the recurrence relation (1.4). Instead, we shall rather study directly the tail of the survival function of $\mathcal{A}_{\mathrm{ex}}$. To do so, we recall the following absolute continuity formula for the normalized excursion of $L$, see [4, Formula (11)]:

$$
\forall s<1, \quad \Lambda_{s} \in \mathcal{F}_{s}, \quad \mathbb{P}_{0,0}^{\uparrow, 1}\left(\Lambda_{s}\right)=c \mathbb{E}_{0}^{\uparrow}\left[1_{\Lambda_{s}} \frac{j_{1-s}^{*}\left(L_{s}\right)}{L_{s}}\right]
$$

where $c$ is a normalization constant and, from Monrad \& Silverstein [20, Formula (3.25)], $j_{1-s}^{*}$ is a measurable function which admits the asymptotics:

$$
j_{1-s}^{*}(x) \underset{x \rightarrow+\infty}{\sim} \gamma(1-s)^{-1 / \alpha} x^{\frac{\alpha}{2(\alpha-1)}} e^{-\eta(1-s)^{-\frac{1}{\alpha-1}} x^{\frac{\alpha}{\alpha-1}}}
$$

where $\gamma$ and $\eta$ are two positive constants. Using this absolute continuity formula, we first deduce that:

$$
\begin{aligned}
\mathbb{P}\left(\mathcal{A}_{\mathrm{ex}}>x\right) & \geq \mathbb{P}_{0,0}^{\uparrow, 1}\left(\int_{1 / 4}^{3 / 4} L_{u}^{\uparrow} d u>x\right) \\
& =c \mathbb{E}_{0}^{\uparrow}\left[\frac{j_{1 / 4}^{*}\left(L_{3 / 4}^{\uparrow}\right)}{L_{3 / 4}^{\uparrow}} 1_{\left\{\int_{1 / 4}^{3 / 4} L_{u}^{\uparrow} d u>x\right\}}\right] \geq c \mathbb{E}_{0}^{\uparrow}\left[\frac{j_{1 / 4}^{*}\left(L_{3 / 4}^{\uparrow}\right)}{L_{3 / 4}^{\uparrow}} 1\left\{\inf _{\frac{1}{4} \leq u \leq \frac{3}{4}} L_{u}^{\uparrow}>2 x\right\}\right] .
\end{aligned}
$$


Applying the Markov property, we further obtain

$$
\begin{aligned}
& \mathbb{P}\left(\mathcal{A}_{\mathrm{ex}}>x\right) \geq c \mathbb{E}_{0}^{\uparrow}\left[\mathbb{E}_{L_{1 / 4}^{\uparrow}}^{\uparrow}\left[\frac{j_{1 / 4}^{*}\left(L_{1 / 2}^{\uparrow}\right)}{L_{1 / 2}^{\uparrow}} 1\left\{\inf _{0 \leq u \leq \frac{1}{2}} L_{u}^{\uparrow}>2 x\right\}\right] 1_{\left\{L_{1 / 4}^{\uparrow}>2 x\right\}}\right] \\
& =c \mathbb{E}_{0}^{\uparrow}\left[\frac{1}{L_{1 / 4}^{\uparrow}} \mathbb{E}_{L_{1 / 4}^{\uparrow}}\left[j_{1 / 4}^{*}\left(L_{1 / 2}\right) 1\left\{\inf _{0 \leq u \leq \frac{1}{2}} L_{u}>2 x\right\}\right] 1_{\left\{L_{1 / 4}^{\uparrow}>2 x\right\}}\right],
\end{aligned}
$$

where we used in the last equality the absolute continuity formula (2.1) for $\mathbb{P}_{L_{1 / 4}}^{\uparrow}$. Now, since $L_{1 / 2} \geq \inf _{0 \leq u \leq \frac{1}{2}} L_{u}>2 x$, we deduce from the asymptotics of $j_{1 / 4}^{*}$ that for $x$ large enough there exist two positive constants $\widetilde{\gamma}$ and $\widetilde{\eta}$ such that

$$
\begin{aligned}
\mathbb{P}\left(\mathcal{A}_{\mathrm{ex}}>x\right) & \geq \widetilde{\gamma} e^{-\widetilde{\eta} x^{\frac{\alpha}{\alpha-1}}} \mathbb{E}_{0}^{\uparrow}\left[\frac{1}{L_{1 / 4}^{\uparrow}} \mathbb{P}_{L_{1 / 4}^{\uparrow}}\left(\inf _{0 \leq u \leq \frac{1}{2}} L_{u}>2 x\right) 1_{\left\{L_{1 / 4}^{\uparrow}>2 x\right\}}\right] . \\
& \geq \widetilde{\gamma} e^{-\widetilde{\eta} x^{\frac{\alpha}{\alpha-1}}} \mathbb{E}_{0}^{\uparrow}\left[\frac{1}{L_{1 / 4}^{\uparrow}} \mathbb{P}_{L_{1 / 4}^{\uparrow}}\left(\inf _{0 \leq u \leq \frac{1}{2}} L_{u}>2 x, L_{1 / 2}<4 x\right) 1_{\left\{4 x>L_{1 / 4}^{\uparrow}>3 x\right\}}\right] \\
& \geq \widetilde{\gamma} e^{-\widetilde{\eta} x^{\frac{\alpha}{\alpha-1}}} \frac{1}{4 x} \mathbb{E}_{0}^{\uparrow}\left[\mathbb{P}_{0}\left(\inf _{0 \leq u \leq \frac{1}{2}} L_{u}>-x, L_{1 / 2}<0\right) 1_{\left\{4 x>L_{1 / 4}^{\uparrow}>3 x\right\}}\right] \\
& =\widetilde{\gamma} e^{-\widetilde{\eta} x^{\frac{\alpha}{\alpha-1}}} \frac{1}{4 x} \mathbb{P}_{0}\left(\inf _{0 \leq u \leq \frac{1}{2}} L_{u}>-x, L_{1 / 2}<0\right) \mathbb{P}_{0}^{\uparrow}\left(4 x>L_{1 / 4}^{\uparrow}>3 x\right)
\end{aligned}
$$

where, in the third inequality, we have used the independent increments property of $L$. The lower bound now follows by taking the logarithm on each side, and using the limits

$$
\mathbb{P}_{0}\left(\inf _{0 \leq u \leq \frac{1}{2}} L_{u}>-x, L_{1 / 2}<0\right) \underset{x \rightarrow+\infty}{\longrightarrow} \mathbb{P}_{0}\left(L_{1 / 2}<0\right)=\frac{1}{\alpha}
$$

and, see Chaumont [4, p. 12],

$$
\mathbb{P}_{0}^{\uparrow}\left(4 x>L_{1 / 4}^{\uparrow}>3 x\right) \underset{x \rightarrow+\infty}{\sim} \kappa x^{-1-\alpha}
$$

for some $\kappa>0$. The lower bound for $\mathbb{E}\left[\mathcal{A}_{\mathrm{ex}}^{n}\right]^{\frac{1}{n}}$ follows then as before from Kasahara's Tauberian theorem of exponential type [11, Theorem 4].

\subsection{Study of some (fractional) negative moments}

As was observed by Flajolet \& Louchard [6] for the classic Airy distribution, one may also compute by induction some specific negative moments. These moments are related to the asymptotic expansion of $\Phi_{\alpha}$ at $\lambda=0$, which is given by its very definition as a series.

Corollary 3.1. Let us set for $n \geq 1$ :

$$
\Delta_{n}=\mathbb{E}\left[\mathcal{A}_{\mathrm{ex}}^{\frac{1-\alpha n}{\alpha+1}}\right] \Gamma\left(\frac{\alpha n-1}{\alpha+1}\right)(1+\alpha)^{-\frac{n+1}{1+\alpha}} .
$$

Then, the sequence $\left(\Delta_{n}\right)$ follows the recurrence relation

$$
\Delta_{n}+\sum_{k=1}^{n-1}\left(\begin{array}{l}
n \\
k
\end{array}\right) \Delta_{n-k} \frac{\Gamma\left(\frac{\alpha}{\alpha+1}\right)}{\Gamma\left(\frac{\alpha-k}{\alpha+1}\right)}=\left(1+\frac{1}{\alpha}\right) \Gamma\left(-\frac{1}{\alpha}\right)\left(\frac{\Gamma\left(\frac{\alpha}{\alpha+1}\right)}{\Gamma\left(\frac{\alpha-1-n}{\alpha+1}\right)}-\frac{\Gamma^{2}\left(\frac{\alpha}{\alpha+1}\right)}{\Gamma\left(\frac{\alpha-1}{\alpha+1}\right) \Gamma\left(\frac{\alpha-n}{\alpha+1}\right)}\right) .
$$


In particular, the first value is given by:

$$
\mathbb{E}\left[\mathcal{A}_{\mathrm{ex}}^{\frac{1-\alpha}{\alpha+1}}\right]=(1+\alpha)^{\frac{3+\alpha}{1+\alpha}} \frac{\Gamma\left(-\frac{1}{\alpha}\right)}{\Gamma\left(\frac{\alpha-1}{\alpha+1}\right)}\left(\frac{\Gamma\left(\frac{\alpha}{\alpha+1}\right)}{\Gamma\left(\frac{\alpha-2}{\alpha+1}\right)}-\frac{\Gamma^{2}\left(\frac{\alpha}{\alpha+1}\right)}{\Gamma^{2}\left(\frac{\alpha-1}{\alpha+1}\right)}\right) .
$$

Proof. We first prove that the negative moments of $\mathcal{A}_{\text {ex }}$ exist. To to so, observe that differentiating $n$ times the formula in Theorem 1, we obtain for $\lambda>0$ :

$$
(-1)^{n-1} \int_{0}^{+\infty} t^{n-1-\frac{1}{\alpha}} e^{-\lambda t} \mathbb{E}\left[e^{-t^{1+\frac{1}{\alpha}} \mathcal{A}_{\mathrm{ex}}}\right] d t=\alpha \Gamma\left(1-\frac{1}{\alpha}\right) \frac{\mathrm{d}^{n}}{\mathrm{~d} \lambda^{n}}\left(\frac{\Phi_{\alpha}^{\prime}(\lambda)}{\Phi_{\alpha}(\lambda)}\right) .
$$

We now let $\lambda \downarrow 0$. Since $\Phi_{\alpha}$ is analytic on $\mathbb{C}$ and $\Phi_{\alpha}(0) \neq 0$, the right-hand side is well-defined for $\lambda=0$. Applying the monotone convergence theorem on the left-hand side, we obtain that

$$
\int_{0}^{+\infty} t^{n-1-\frac{1}{\alpha}} \mathbb{E}\left[e^{-t^{1+\frac{1}{\alpha}}} \mathcal{A}_{\mathrm{ex}}\right] d t=\frac{\alpha}{\alpha+1} \Gamma\left(\frac{\alpha n-1}{\alpha+1}\right) \mathbb{E}\left[\mathcal{A}_{\mathrm{ex}}^{\frac{\alpha n-1}{\alpha+1}}\right]
$$

which is therefore also finite. Next, to get the recurrence formula, we go back to Theorem 1.1 and write the Taylor expansion of $1-e^{-\lambda t}$. Multiplying then both sides by $\Phi_{\alpha}$, we obtain

$$
\begin{aligned}
\Phi_{\alpha}(\lambda)\left(\frac{\alpha}{\alpha+1} \sum_{k=1}^{n} \frac{(-1)^{k+1}}{k !} \lambda^{k} \mathbb{E}\left[\mathcal{A}_{\mathrm{ex}}^{\frac{1-\alpha k}{\alpha+1}}\right] \Gamma\left(\frac{\alpha k-1}{\alpha+1}\right)\right. \\
\left.+(-1)^{n} \frac{\lambda^{n+1}}{n !} \int_{0}^{+\infty} t^{n-\frac{1}{\alpha}} \int_{0}^{1}(1-s)^{n} e^{-\lambda t s} \mathbb{E}\left[e^{-t^{1+\frac{1}{\alpha}}} \mathcal{A}_{\mathrm{ex}}\right] d t d s\right) \\
=\Gamma\left(-\frac{1}{\alpha}\right)\left(\Phi_{\alpha}^{\prime}(\lambda)+(1+\alpha)^{\frac{1}{1+\alpha}} \frac{\Gamma\left(\frac{\alpha}{\alpha+1}\right)}{\Gamma\left(\frac{\alpha-1}{\alpha+1}\right)} \Phi_{\alpha}(\lambda)\right) .
\end{aligned}
$$

The result now follows by computing the Cauchy product on the left-hand side, and by identifying both expansions.

\section{The area under a spectrally positive stable meander}

\subsection{Proof of Theorem 1.3: the double Laplace transform}

We shall work here with a Laplace-Fourier transform to avoid integrability problems. Observe first that by analytic continuation, Proposition 2.1 remains valid for $\lambda$ and $\mu$ in the open set $\{z \in \mathbb{C} ; \Re(z)>0\}$. Letting $\mu \rightarrow i$, we thus obtain:

$$
\mathbb{E}_{z}\left[e^{-\lambda T_{0}-i \int_{0}^{T_{0}} L_{u} d u}\right]=\frac{\Phi_{\alpha}\left(i^{\frac{1}{1+\alpha}}\left(z+\frac{\lambda}{i}\right)\right)}{\Phi_{\alpha}\left(i^{-\frac{\alpha}{1+\alpha}} \lambda\right)} .
$$

Applying then the Markov property, we have for $z>0$ and $\xi$ such that $\Re(\xi)>0$ :

$$
\begin{aligned}
& \int_{0}^{+\infty} e^{-\xi t} \mathbb{E}_{z}\left[e^{-i \int_{0}^{t} L_{u} d u} 1_{\left\{T_{0}>t\right\}}\right] d t \\
& =\int_{0}^{+\infty} e^{-\xi t} \mathbb{E}_{z}\left[e^{-i \int_{0}^{t} L_{u} d u}\right] d t-\int_{0}^{+\infty} e^{-\xi t} \mathbb{E}_{z}\left[e^{-i \int_{0}^{t} L_{u} d u} 1_{\left\{T_{0} \leq t\right\}}\right] d t \\
& =\int_{0}^{+\infty} e^{-\xi t} \mathbb{E}_{z}\left[e^{-i \int_{0}^{t} L_{u} d u}\right] d t-\mathbb{E}_{z}\left[e^{-\xi T_{0}-i \int_{0}^{T_{0}} L_{u} d u}\right] \int_{0}^{+\infty} e^{-\xi t} \mathbb{E}_{0}\left[e^{-i \int_{0}^{t} L_{u} d u}\right] d t \\
& =\int_{0}^{+\infty} e^{-\xi t} \mathbb{E}_{0}\left[e^{-i z t-i \int_{0}^{t} L_{u} d u}\right] d t-\frac{\Phi_{\alpha}\left(i^{\frac{1}{1+\alpha}}\left(z+\frac{\xi}{i}\right)\right)}{\Phi_{\alpha}\left(i^{-\frac{\alpha}{1+\alpha}} \xi\right)} \int_{0}^{+\infty} e^{-\xi t} \mathbb{E}_{0}\left[e^{-i \int_{0}^{t} L_{u} d u}\right] d t .
\end{aligned}
$$


We now divide this equality by $z$ and let $z \downarrow 0$. Using (3.3), the scaling property and the definition of the meander, the left-hand side converges towards

$$
\begin{aligned}
& \frac{1}{z} \int_{0}^{+\infty} e^{-\xi t} \mathbb{E}_{z}\left[e^{-i \int_{0}^{t} L_{u} d u} \mid T_{0}>t\right] \mathbb{P}_{z}\left(T_{0}>t\right) d t \\
& \underset{z \downarrow 0}{\longrightarrow} \frac{1}{\Gamma\left(1-\frac{1}{\alpha}\right)} \int_{0}^{+\infty} e^{-\xi t} \mathbb{E}\left[e^{-i t^{1+1 / \alpha} \mathcal{A}_{\mathrm{m}}}\right] t^{-1 / \alpha} d t
\end{aligned}
$$

while the right-hand side yields

$$
-\int_{0}^{+\infty} i t e^{-\xi t} \mathbb{E}_{0}\left[e^{-i \int_{0}^{t} L_{u} d u}\right] d t-i^{\frac{1}{1+\alpha}} \frac{\left.\Phi_{\alpha}^{\prime}\left(i^{-\frac{\alpha}{1+\alpha}} \xi\right)\right)}{\Phi_{\alpha}\left(i^{-\frac{\alpha}{1+\alpha}} \xi\right)} \int_{0}^{+\infty} e^{-\xi t} \mathbb{E}_{0}\left[e^{-i \int_{0}^{t} L_{u} d u}\right] d t
$$

Let us set, to simplify the notation:

$$
F_{\alpha}(\xi)=\int_{0}^{+\infty} e^{-\xi t} \mathbb{E}_{0}\left[e^{-i \int_{0}^{t} L_{u} d u}\right] d t .
$$

From (1.1), we deduce that for $\xi \in \mathbb{C}$ :

$$
\begin{aligned}
F_{\alpha}(\xi) & =\int_{0}^{+\infty} e^{-\xi t} \exp \left(e^{\frac{i \pi}{2} \alpha} \frac{t^{1+\alpha}}{1+\alpha}\right) d t \\
& =\frac{1}{1+\alpha} \sum_{n=0}^{+\infty} \frac{(-1)^{n}}{n !} \xi^{n} \Gamma\left(\frac{n+1}{\alpha+1}\right) e^{\frac{i \pi(2-\alpha)}{2} \frac{n+1}{\alpha+1}}(1+\alpha)^{\frac{n+1}{\alpha+1}} \\
& =\pi i^{-\frac{\alpha}{1+\alpha}}\left(\Psi_{\alpha}\left(i^{-\frac{\alpha}{1+\alpha}} \xi\right)+i \Phi_{\alpha}\left(i^{-\frac{\alpha}{1+\alpha}} \xi\right)\right)
\end{aligned}
$$

which shows that $\Phi_{\alpha}$ and $\Psi_{\alpha}$ are closely related to the integral of $L$. Notice also that

$$
-\int_{0}^{+\infty} i t e^{-\xi t} \mathbb{E}_{0}\left[e^{-i \int_{0}^{t} L_{u} d u}\right] d t=i F_{\alpha}^{\prime}(\xi) .
$$

Plugging (4.3) and (4.4) in (4.1) then yields the formula

$$
\begin{aligned}
\int_{0}^{+\infty} e^{-\xi t} \mathbb{E}\left[e^{-i t^{1+\frac{1}{\alpha}}} \mathcal{A}_{\mathrm{me}}\right] t^{-\frac{1}{\alpha}} d t \\
\quad=\pi \Gamma\left(1-\frac{1}{\alpha}\right) i^{\frac{1-\alpha}{1+\alpha}} \frac{\Psi_{\alpha}^{\prime}\left(i^{-\frac{\alpha}{1+\alpha}} \xi\right) \Phi_{\alpha}\left(i^{-\frac{\alpha}{1+\alpha}} \xi\right)-\Phi_{\alpha}^{\prime}\left(i^{-\frac{\alpha}{1+\alpha}} \xi\right) \Psi_{\alpha}\left(i^{-\frac{\alpha}{1+\alpha}} \xi\right)}{\Phi_{\alpha}\left(i^{-\frac{\alpha}{1+\alpha}} \xi\right)} .
\end{aligned}
$$

Finally, applying Cauchy's integral theorem with the curve $\{t ; 0 \leq t \leq R\} \cup\left\{t i^{-\frac{\alpha}{1+\alpha}} ; 0 \leq\right.$ $t \leq R\} \cup\left\{R e^{i \theta}, 0 \leq \theta \leq-\frac{\pi \alpha}{2(1+\alpha))}\right\}$ and letting $R \rightarrow+\infty$, we deduce that

$$
\int_{0}^{+\infty} e^{-\xi t} e^{-i t^{1+\frac{1}{\alpha}} \mathcal{A}_{\mathrm{me}}} t^{-\frac{1}{\alpha}} d t=i^{\frac{1-\alpha}{1+\alpha}} \int_{0}^{+\infty} e^{-\xi i^{-\frac{\alpha}{1+\alpha}}} e^{-t^{1+\frac{1}{\alpha}} \mathcal{A}_{\mathrm{me}}} t^{-\frac{1}{\alpha}} d t
$$

and the result follows by taking the expectation on both sides, applying Fubini's theorem, and then replacing $\xi \in\{z \in \mathbb{C}, \Re(z)>0\}$ by $\lambda i^{\frac{\alpha}{1+\alpha}}$ with $\lambda \in(0,+\infty)$.

\subsection{Proof of Theorem 1.3: first moment and asymptotics}

To simplify the following computation, we set

$$
H_{\alpha}(\lambda)=\pi \frac{\Psi_{\alpha}^{\prime}(\lambda) \Phi_{\alpha}(\lambda)-\Phi_{\alpha}^{\prime}(\lambda) \Psi_{\alpha}(\lambda)}{\Phi_{\alpha}(\lambda)} .
$$


The area under a spectrally positive stable excursion

We start by computing the expectation of $\mathcal{A}_{\text {me }}$. Plugging the decomposition

$$
\mathbb{E}\left[e^{-t^{1+\frac{1}{\alpha}}} \mathcal{A}_{\mathrm{me}}\right]=1-\int_{0}^{+\infty} t^{1+\frac{1}{\alpha}} e^{-z t^{1+\frac{1}{\alpha}}} \mathbb{P}\left(\mathcal{A}_{\mathrm{me}}>z\right) d z
$$

into Formula (1.5), we first deduce that

$$
\int_{0}^{+\infty} e^{-\lambda t} t \int_{0}^{+\infty} e^{-z t^{1+\frac{1}{\alpha}}} \mathbb{P}\left(\mathcal{A}_{\mathrm{me}}>z\right) d z d t=\Gamma\left(1-\frac{1}{\alpha}\right)\left(\frac{1}{\lambda^{1-\frac{1}{\alpha}}}-H_{\alpha}(\lambda)\right) .
$$

We then integrate this relation with respect to $\lambda$ on $(\xi,+\infty)$. This gives, using the Fubini-Tonelli theorem:

$$
\int_{0}^{+\infty} e^{-\xi t} \int_{0}^{+\infty} e^{-z t^{1+\frac{1}{\alpha}}} \mathbb{P}\left(\mathcal{A}_{\mathrm{me}}>z\right) d z d t=\Gamma\left(1-\frac{1}{\alpha}\right) \int_{\xi}^{+\infty}\left(\frac{1}{\lambda^{1-\frac{1}{\alpha}}}-H_{\alpha}(\lambda)\right) d \lambda .
$$

Finally, the change of variable $s=\xi t$, yields:

$$
\int_{0}^{+\infty} e^{-s} \int_{0}^{+\infty} e^{-z\left(\frac{s}{\xi}\right)^{1+\frac{1}{\alpha}}} \mathbb{P}\left(\mathcal{A}_{\mathrm{me}}>z\right) d z d s=\Gamma\left(1-\frac{1}{\alpha}\right) \xi \int_{\xi}^{+\infty}\left(\frac{1}{\lambda^{1-\frac{1}{\alpha}}}-H_{\alpha}(\lambda)\right) d \lambda .
$$

Taking the limit as $\xi \rightarrow+\infty$, we deduce from the monotone convergence theorem that the left-hand side converge towards $\mathbb{E}\left[\mathcal{A}_{\text {me }}\right]$. To compute the limit of the right-hand side, we need to study the asymptotics of $H_{\alpha}$. Since the asymptotics of $\Phi_{\alpha}$ and $\Phi_{\alpha}^{\prime}$ are given in Proposition A.2 in the Appendix, it only remains to study those of $\Psi_{\alpha}$ and $\Psi_{\alpha}^{\prime}$. To this end, observe from (4.3) that

$$
\Psi_{\alpha}(\lambda)=\frac{i^{\frac{\alpha}{1+\alpha}}}{\pi} F_{\alpha}\left(\lambda i^{\frac{\alpha}{1+\alpha}}\right)-i \Phi_{\alpha}(\lambda)
$$

Applying Watson's lemma, we deduce from the definition (4.2) of $F_{\alpha}$ that

$$
F_{\alpha}\left(\lambda i^{\frac{\alpha}{1+\alpha}}\right) \underset{\lambda \rightarrow+\infty}{\sim} i^{-\frac{\alpha}{1+\alpha}} \sum_{n=0}^{+\infty} \frac{1}{n !} \frac{\Gamma(1+(1+\alpha) n)}{(1+\alpha)^{n}} \lambda^{-n(1+\alpha)-1}
$$

which implies, since $\Phi_{\alpha}$ decreases exponentially fast, the asymptotic expansion

$$
\Psi_{\alpha}(\lambda) \underset{\lambda \rightarrow+\infty}{\sim} \frac{1}{\pi} \sum_{n=0}^{+\infty} \frac{1}{n !} \frac{\Gamma(1+(1+\alpha) n)}{(1+\alpha)^{n}} \lambda^{-n(1+\alpha)-1}
$$

and similarly

$$
\Psi_{\alpha}^{\prime}(\lambda) \underset{\lambda \rightarrow+\infty}{\sim}-\frac{1}{\pi} \sum_{n=0}^{+\infty} \frac{1}{n !} \frac{\Gamma(2+(1+\alpha) n)}{(1+\alpha)^{n}} \lambda^{-n(1+\alpha)-2} .
$$

Therefore, going back to the definition of $H_{\alpha}$, we deduce that

$$
H_{\alpha}(\lambda) \underset{\lambda \rightarrow+\infty}{=}\left(\lambda^{\frac{1}{\alpha}-1}-\frac{\alpha+1}{2 \alpha} \lambda^{-2}+\Gamma(1+\alpha) \lambda^{-2-\alpha+\frac{1}{\alpha}}\right)+\mathrm{o}\left(\lambda^{-2-\alpha+\frac{1}{\alpha}}\right) .
$$

As a consequence, we obtain the limit

$$
\lim _{\xi \rightarrow+\infty} \xi \int_{\xi}^{+\infty}\left(\frac{1}{\lambda^{1-\frac{1}{\alpha}}}-H_{\alpha}(\lambda)\right) d \lambda=\lim _{\xi \rightarrow+\infty} \xi \int_{\xi}^{+\infty} \frac{\alpha+1}{2 \alpha} \lambda^{-2} d \lambda=\frac{\alpha+1}{2 \alpha}
$$

from which we deduce that the first moment is finite and equals

$$
\mathbb{E}\left[\mathcal{A}_{\mathrm{me}}\right]=\Gamma\left(1-\frac{1}{\alpha}\right) \frac{\alpha+1}{2 \alpha} .
$$


We now compute the asymptotics of $\mathbb{P}\left(\mathcal{A}_{\text {me }}>x\right)$. Integrating by parts, we first write the decomposition:

$$
\int_{0}^{+\infty} e^{-z t^{1+\frac{1}{\alpha}}} \mathbb{P}\left(\mathcal{A}_{\mathrm{me}}>z\right) d z=\mathbb{E}\left[\mathcal{A}_{\mathrm{me}}\right]-\int_{0}^{+\infty} t^{1+\frac{1}{\alpha}} e^{-z t^{1+\frac{1}{\alpha}}} \int_{z}^{+\infty} \mathbb{P}\left(\mathcal{A}_{\mathrm{me}}>u\right) d u d z
$$

Plugging this formula into (4.5) and replacing $\mathbb{E}\left[\mathcal{A}_{\mathrm{me}}\right]$ by its value then yields:

$$
\begin{aligned}
\int_{0}^{+\infty} e^{-\lambda t} t \int_{0}^{+\infty} t^{1+\frac{1}{\alpha}} e^{-z t^{1+\frac{1}{\alpha}}} \int_{z}^{+\infty} \mathbb{P}\left(\mathcal{A}_{\mathrm{me}}>u\right) d u d z d t & \\
& =\Gamma\left(1-\frac{1}{\alpha}\right)\left(\frac{\alpha+1}{2 \alpha} \lambda^{-2}-\lambda^{\frac{1}{\alpha}-1}+H_{\alpha}(\lambda)\right) .
\end{aligned}
$$

Integrating as before in $\lambda$ on $(\xi,+\infty)$ we obtain:

$$
\begin{aligned}
\int_{0}^{+\infty} e^{-\xi t} \int_{0}^{+\infty} \int_{0}^{+\infty} t^{1+\frac{1}{\alpha}} e^{-z t^{1+\frac{1}{\alpha}}} \int_{z}^{+\infty} \mathbb{P}\left(\mathcal{A}_{\mathrm{me}}>u\right) d u d z d t \\
=\Gamma\left(1-\frac{1}{\alpha}\right) \int_{\xi}^{+\infty}\left(\frac{\alpha+1}{2 \alpha} \lambda^{-2}-\lambda^{\frac{1}{\alpha}-1}+H_{\alpha}(\lambda)\right) d \lambda
\end{aligned}
$$

and the right-hand side is equivalent to:

$$
\int_{\xi}^{+\infty}\left(\frac{\alpha+1}{2 \alpha} \lambda^{-2}-\lambda^{\frac{1}{\alpha}-1}+H_{\alpha}(\lambda)\right) d \lambda \underset{\xi \rightarrow+\infty}{\sim} \frac{\Gamma(1+\alpha)}{1+\alpha-\frac{1}{\alpha}} \xi^{-1-\alpha+\frac{1}{\alpha}} .
$$

Applying Karamata's Tauberian theorem in (4.7), we thus deduce that

$$
t^{1+\frac{1}{\alpha}} \int_{0}^{+\infty} \int_{0}^{+\infty} e^{-z t^{1+\frac{1}{\alpha}}} \int_{z}^{+\infty} \mathbb{P}\left(\mathcal{A}_{\mathrm{me}}>u\right) d u d z \underset{t \rightarrow 0}{\sim} \frac{\Gamma\left(1-\frac{1}{\alpha}\right) \Gamma(1+\alpha)}{\Gamma\left(2+\alpha-\frac{1}{\alpha}\right)} t^{\alpha-\frac{1}{\alpha}} .
$$

Note that the function on the left-hand side is increasing, as may be easily seen by performing the change of variable $z t^{1+\frac{1}{\alpha}}=x$. Another application of Karamata's Tauberian theorem yields

$$
\int_{z}^{+\infty} \mathbb{P}\left(\mathcal{A}_{\mathrm{me}}>u\right) d u \underset{z \rightarrow+\infty}{\sim} \frac{\Gamma\left(1-\frac{1}{\alpha}\right) \Gamma(1+\alpha)}{\Gamma\left(2+\alpha-\frac{1}{\alpha}\right) \Gamma(2-\alpha)} z^{1-\alpha}
$$

Since the function on the left-hand side is concave, we may differentiate this relation to finally obtain:

$$
\mathbb{P}\left(\mathcal{A}_{\mathrm{me}}>z\right) \underset{z \rightarrow+\infty}{\sim} \frac{(\alpha-1) \Gamma\left(1-\frac{1}{\alpha}\right) \Gamma(1+\alpha)}{\Gamma\left(2+\alpha-\frac{1}{\alpha}\right) \Gamma(2-\alpha)} z^{-\alpha}
$$

which is the announced asymptotics.

\section{The area under $L$ conditioned to stay positive}

\subsection{Proof of Theorem 1.5: the double Laplace transform}

We proceed as for the meander. Applying the Markov property, we first have for $z>0$ and $\xi$ such that $\Re(\xi)>0$ :

$$
\begin{aligned}
& \int_{0}^{+\infty} e^{-\xi t} \mathbb{E}_{z}\left[L_{t} e^{-i \int_{0}^{t} L_{u} d u} 1_{\left\{T_{0}>t\right\}}\right] d t \\
& =\int_{0}^{+\infty} e^{-\xi t} \mathbb{E}_{z}\left[L_{t} e^{-i \int_{0}^{t} L_{u} d u}\right] d t-\frac{\Phi_{\alpha}\left(i^{\frac{1}{1+\alpha}}\left(z+\frac{\xi}{i}\right)\right)}{\Phi_{\alpha}\left(i^{-\frac{\alpha}{1+\alpha}} \xi\right)} \int_{0}^{+\infty} e^{-\xi t} \mathbb{E}_{0}\left[L_{t} e^{-i \int_{0}^{t} L_{u} d u}\right] d t
\end{aligned}
$$


Dividing both sides by $z$ and letting $z \downarrow 0$ then yields by definition of $\mathbb{P}_{0}^{\uparrow}$ :

$$
\begin{aligned}
& \int_{0}^{+\infty} e^{-\xi t} \mathbb{E}_{0}^{\uparrow}\left[e^{-i \int_{0}^{t} L_{u} d u}\right] d t \\
= & \int_{0}^{+\infty} e^{-\xi t} \mathbb{E}_{0}\left[\left(1-i t L_{t}\right) e^{-i \int_{0}^{t} L_{u} d u}\right] d t-i^{\frac{1}{1+\alpha}} \frac{\Phi_{\alpha}^{\prime}\left(i^{-\frac{\alpha}{1+\alpha}} \xi\right)}{\Phi_{\alpha}\left(i^{-\frac{\alpha}{1+\alpha}} \xi\right)} \int_{0}^{+\infty} e^{-\xi t} \mathbb{E}_{0}\left[L_{t} e^{-i \int_{0}^{t} L_{u} d u}\right] d t .
\end{aligned}
$$

Observe next that, integrating by parts the definition (4.2) of $F_{\alpha}$, we also have

$$
F_{\alpha}(\xi)=\frac{1}{\xi}-\frac{i}{\xi} \int_{0}^{+\infty} e^{-\xi t} \mathbb{E}_{0}\left[L_{t} e^{-i \int_{0}^{t} L_{u} d u}\right] d t
$$

As a consequence, we deduce from (4.3) that

$$
\begin{aligned}
& \int_{0}^{+\infty} e^{-\xi t} \mathbb{E}_{0}^{\uparrow}\left[e^{-i t^{1+\frac{1}{\alpha}} \int_{0}^{1} L_{u} d u}\right] d t \\
& =-\xi F_{\alpha}^{\prime}(\xi)-i^{-\frac{\alpha}{1+\alpha}} \frac{\Phi_{\alpha}^{\prime}\left(i^{-\frac{\alpha}{1+\alpha}} \xi\right)}{\Phi_{\alpha}\left(i^{-\frac{\alpha}{1+\alpha}} \xi\right)}\left(1-\xi F_{\alpha}(\xi)\right) \\
& =\pi i^{-\frac{2 \alpha}{1+\alpha}} \xi \frac{\Phi_{\alpha}^{\prime}\left(i^{-\frac{\alpha}{1+\alpha}} \xi\right) \Psi_{\alpha}\left(i^{-\frac{\alpha}{1+\alpha}} \xi\right)-\Psi_{\alpha}^{\prime}\left(i^{-\frac{\alpha}{1+\alpha}} \xi\right) \Phi_{\alpha}\left(i^{-\frac{\alpha}{1+\alpha}} \xi\right)}{\Phi_{\alpha}\left(i^{-\frac{\alpha}{1+\alpha}} \xi\right)}-i^{-\frac{\alpha}{1+\alpha}} \frac{\Phi_{\alpha}^{\prime}\left(i^{-\frac{\alpha}{1+\alpha}} \xi\right)}{\Phi_{\alpha}\left(i^{-\frac{\alpha}{1+\alpha}} \xi\right)} .
\end{aligned}
$$

As before, Cauchy's integral theorem shows that

$$
\int_{0}^{+\infty} e^{-\xi t} e^{-i t^{1+\frac{1}{\alpha}} \int_{0}^{1} L_{u} d u} d t=i^{-\frac{\alpha}{1+\alpha}} \int_{0}^{+\infty} e^{-\xi i^{-\frac{\alpha}{1+\alpha}} t} e^{-t^{1+\frac{1}{\alpha}} \int_{0}^{1} L_{u} d u} d t
$$

and the result follows by taking expectations and replacing $\xi$ by $\lambda i^{\frac{\alpha}{1+\alpha}}$ with $\lambda>0$.

\subsection{Proof of Theorem 1.5: asymptotics}

The asymptotics of the tail of $\mathcal{A}^{\uparrow}$ is easy to obtain as we can directly apply Karamata's Tauberian theorem. Indeed, since we are dealing with monotone integrands, there is the asymptotics for $\alpha<2$ :

$$
\int_{0}^{+\infty} e^{-\lambda t} \mathbb{E}\left[1-e^{-t^{1+\frac{1}{\alpha}} \mathcal{A}^{\uparrow}}\right] d t=\frac{1}{\lambda}+\lambda H_{\alpha}(\lambda)+\frac{\Phi_{\alpha}^{\prime}(\lambda)}{\Phi_{\alpha}(\lambda)} \underset{\lambda \rightarrow+\infty}{\sim} \frac{\Gamma(1+\alpha)}{\lambda^{1+\alpha-\frac{1}{\alpha}}}
$$

which implies that

$$
\mathbb{E}\left[1-e^{-t^{1+\frac{1}{\alpha}}} \mathcal{A}^{\uparrow}\right] \underset{t \rightarrow 0}{\sim} \frac{\Gamma(1+\alpha)}{\Gamma\left(1+\alpha-\frac{1}{\alpha}\right)} t^{\alpha-\frac{1}{\alpha}}
$$

which in turn implies that

$$
\mathbb{P}\left(\mathcal{A}^{\uparrow}>x\right) \underset{x \rightarrow+\infty}{\sim} \frac{\Gamma(1+\alpha)}{\Gamma\left(1+\alpha-\frac{1}{\alpha}\right) \Gamma(2-\alpha)} x^{1-\alpha} .
$$

\section{A Appendix on M-Wright's functions}

We gather and prove in this section several useful formulae for the M-Wright function $\Phi_{\alpha}$ and its derivative.

\section{A.1 The integral representation}

Following the notation of [7], the classic Wright function is defined by:

$$
\phi(\rho, \beta ; z)=\sum_{k=0}^{+\infty} \frac{z^{k}}{k ! \Gamma(\rho k+\beta)}, \quad \rho>-1, \beta \in \mathbb{C} .
$$


A special case of this function is obtained when $\rho=\beta-1=-\frac{1}{1+\alpha}$, for which, applying the compensation formula for the Gamma function, we obtain:

$$
\begin{aligned}
\Phi_{\alpha}(x) & =(1+\alpha)^{-\frac{\alpha}{1+\alpha}} \phi\left(-\frac{1}{1+\alpha}, \frac{\alpha}{1+\alpha} ;-x(1+\alpha)^{\frac{1}{1+\alpha}}\right) \\
& =\frac{1}{\pi} \sum_{n=0}^{+\infty} \frac{(-1)^{n}}{n !} \Gamma\left(\frac{1+n}{1+\alpha}\right) \sin \left(\pi \frac{1+n}{\alpha+1}\right)(1+\alpha)^{\frac{n-\alpha}{1+\alpha}} x^{n}
\end{aligned}
$$

which is nowadays referred in the literature as an M-Wright function. This function is also closely related to the probability density of the positive stable distribution of parameter $\frac{1}{\alpha+1}$, see Sato [23, p. 88]. To get an integral expression, recall the formulae, since $\alpha>1$ :

$$
\int_{0}^{\infty e^{ \pm i \pi /(1+\alpha)}} z^{n} e^{\frac{z^{1+\alpha}}{1+\alpha}} d z=e^{ \pm i \frac{\pi(n+1)}{1+\alpha}}(1+\alpha)^{\frac{n-\alpha}{\alpha+1}} \Gamma\left(\frac{n+1}{\alpha+1}\right)
$$

which implies

$$
\begin{aligned}
\Phi_{\alpha}(x) & =\frac{1}{2 \pi i} \sum_{n=0}^{+\infty} \frac{(-1)^{n}}{n !} x^{n}\left(\int_{0}^{\infty e^{i \pi /(1+\alpha)}} z^{n} e^{\frac{z^{1+\alpha}}{1+\alpha}} d z-\int_{0}^{\infty e^{-i \pi /(1+\alpha)}} z^{n} e^{\frac{z^{1+\alpha}}{1+\alpha}} d z\right) \\
& =\frac{1}{2 \pi i} \int_{\infty e^{-i \pi /(1+\alpha)}}^{\infty e^{i \pi /(1+\alpha)}} e^{\frac{z^{1+\alpha}}{1+\alpha}-z x} d z .
\end{aligned}
$$

Applying Cauchy's integral theorem, we may deform the path of integration to pass by the imaginary axis, and thus obtain the integral representation:

$$
\Phi_{\alpha}(x)=\frac{1}{\pi} \int_{0}^{+\infty} e^{-\sin \left(\frac{\pi \alpha}{2}\right) \frac{z^{1+\alpha}}{1+\alpha}} \cos \left(\cos \left(\frac{\pi \alpha}{2}\right) \frac{z^{1+\alpha}}{1+\alpha}-z x\right) d z .
$$

\section{A.2 Asymptotic expansion of $\Phi_{\alpha}$ and $\Phi_{\alpha}^{\prime}$}

We now study the asymptotics of $\Phi_{\alpha}(x)$ as $x \rightarrow+\infty$. A general (theoretical) asymptotic expansion for $\phi$ was computed by Wright [26] (see also [7, Theorem 2.1.3]), but it seems difficult to extract from his formula an explicit expression for the coefficients. This will be our objective here.

Recall the following definition of the coefficients $\left(c_{p}^{(\alpha)}\right)$ :

$$
c_{0}^{(\alpha)}=1 \quad \text { and } \quad c_{p}^{(\alpha)}=\frac{1}{(2 p) ! \sqrt{\pi}}\left(\frac{2}{\alpha}\right)^{p} \sum_{k=1}^{2 p} B_{2 p, k} \Gamma\left(p+k+\frac{1}{2}\right)(2(\alpha-1))^{k}, \quad p \geq 1
$$

where the sequence $\left(B_{n, k}\right)$ is defined for any $n \geq 1$ by

$$
B_{n, 1}=\frac{(2-\alpha)_{n-1}}{(n+1)(n+2)} \quad \text { and } \quad B_{n, k+1}=\frac{1}{k+1} \sum_{l=k}^{n-1}\left(\begin{array}{l}
n \\
l
\end{array}\right) B_{n-l, 1} \times B_{l, k} \quad k \geq 1 .
$$

We start with a simple lemma.

Lemma A.1. For any $k \geq 1$, there is the upper bound:

$$
\forall n \geq 1, \quad \frac{B_{n, k}}{n !} \leq \frac{\left(B_{\infty}\right)^{k}}{k !} \leq \frac{(1 / 4)^{k}}{k !} \quad \text { where } \quad B_{\infty}=\sum_{n=1}^{+\infty} \frac{B_{n, 1}}{n !} .
$$

Proof. We prove the first inequality by iteration on $k$. For $k=1$, this is obviously true since $\frac{B_{n, 1}}{n !} \leq B_{\infty}$. Assume now that (A.2) holds for all the integers up to some $k$. Then, 
from the definition (1.2) of the sequence $\left(B_{n, k}\right)$ (which is recalled above), we have

$$
\frac{B_{n, k+1}}{n !}=\frac{1}{k+1} \sum_{l=k}^{n-1} \frac{B_{n-l, 1}}{(n-l) !} \frac{B_{l, k}}{l !} \leq \frac{\left(B_{\infty}\right)^{k}}{(k+1) !} \sum_{l=k}^{n-1} \frac{B_{n-l, 1}}{(n-l) !} \leq \frac{\left(B_{\infty}\right)^{k+1}}{(k+1) !}
$$

which proves the first inequality. The second one follows from the fact that $(2-\alpha)_{n-1} \leq$ $(n-1)$ ! for all $n \geq 1$, hence, going back to the definition of the sequence $\left(B_{n, 1}\right)$,

$$
B_{\infty} \leq \sum_{n=1}^{+\infty} \frac{1}{n(n+1)(n+2)}=\frac{1}{4}
$$

We may now compute the asymptotic expansion of $\Phi_{\alpha}$ and $\Phi_{\alpha}^{\prime}$.

Proposition A.2. We have the asymptotic expansions as $x \rightarrow+\infty$ :

$$
\Phi_{\alpha}(x) \underset{x \rightarrow+\infty}{\sim} \frac{1}{\sqrt{2 \pi \alpha}} x^{\frac{1-\alpha}{2 \alpha}} e^{-\frac{\alpha}{\alpha+1} x^{1+1 / \alpha}} \sum_{p=0}^{+\infty}(-1)^{p} c_{p}^{(\alpha)} x^{-p(1+1 / \alpha)}
$$

and

$$
\Phi_{\alpha}^{\prime}(x) \underset{x \rightarrow+\infty}{\sim} \frac{1}{\sqrt{2 \pi \alpha}} x^{\frac{3-\alpha}{2 \alpha}} e^{-\frac{\alpha}{\alpha+1} x^{1+1 / \alpha}} \sum_{p=0}^{+\infty}(-1)^{p+1} d_{p}^{(\alpha)} x^{-p(1+1 / \alpha)}
$$

where $d_{0}^{(\alpha)}=1$ and for $p \geq 1$ :

$$
d_{p}^{(\alpha)}=c_{p}^{(\alpha)}-c_{p-1}^{(\alpha)}\left(\frac{(2 p-1)(\alpha+1)-2}{2 \alpha}\right) .
$$

Proof. Since these asymptotics are already known for the Airy function, we shall assume in the following that $\alpha<2$. We start with the asymptotic expansion of $\Phi_{\alpha}$. Coming back to the formula (A.1), we may write, using the change of variable $z=x^{\frac{1}{\alpha}} y$ :

$$
\begin{aligned}
\Phi_{\alpha}(x) & =\frac{x^{\frac{1}{\alpha}}}{2 \pi} \int_{0}^{+\infty} e^{i x^{1+\frac{1}{\alpha}}\left(e^{i \frac{\pi \alpha}{2}} \frac{y^{1+\alpha}}{1+\alpha}-y\right)} d y+\frac{x^{\frac{1}{\alpha}}}{2 \pi} \int_{0}^{+\infty} e^{i x^{1+\frac{1}{\alpha}}\left(e^{i \pi-i \frac{\pi \alpha}{2}} \frac{y^{1+\alpha}}{1+\alpha}-y\right)} d y \\
& =\frac{x^{\frac{1}{\alpha}}}{2 \pi} \int_{-\infty}^{+\infty} e^{i x^{1+\frac{1}{\alpha}}\left(e^{i \frac{\pi \alpha}{2}} \frac{y^{1+\alpha}}{1+\alpha}-y\right)} d y
\end{aligned}
$$

where, for $y<0$, we take $\arg (y)=-\pi$. Applying Cauchy's integral theorem, we first deform the path of integration to pass through the line $\{z \in \mathbb{C}, \Im(z)=-1\}$ :

$$
\Phi_{\alpha}(x)=\frac{x^{\frac{1}{\alpha}}}{2 \pi} \int_{-\infty}^{+\infty} e^{i x^{1+\frac{1}{\alpha}}\left(i^{\alpha} \frac{(u-i)^{1+\alpha}}{1+\alpha}-(u-i)\right)} d u
$$

Recall next the following Taylor expansion:

$$
\frac{(u-i)^{1+\alpha}}{1+\alpha}=\frac{(-i)^{1+\alpha}}{1+\alpha}+u(-i)^{\alpha}+\frac{u^{2}}{2} \alpha(-i)^{\alpha-1}+\alpha(\alpha-1) \frac{u^{3}}{2} \int_{0}^{1}(1-t)^{2}(t u-i)^{\alpha-2} d t
$$

as well as Euler's integral formula for the hypergeometric function ${ }_{2} F_{1}$ :

$$
\int_{0}^{1}(1-t)^{2}(t u-i)^{\alpha-2} d t=\frac{1}{3}(-i)^{\alpha-2}{ }_{2} F_{1}\left[\begin{array}{rr}
2-\alpha & 1 \\
4 & ;-i u
\end{array}\right] .
$$

Setting $\xi=x^{1+1 / \alpha}$ and making the change of variable $u \sqrt{\xi}=z$, we further obtain

$$
\Phi_{\alpha}\left(\xi^{\frac{\alpha}{\alpha+1}}\right)=\frac{1}{2 \pi} \xi^{\frac{1}{1+\alpha}-\frac{1}{2}} e^{-\frac{\alpha}{\alpha+1} \xi} \int_{-\infty}^{+\infty} e^{-\alpha \frac{z^{2}}{2}+z^{2} \alpha(\alpha-1) \varphi\left(\frac{z}{\sqrt{\xi}}\right)} d z
$$


where

$$
\varphi\left(\frac{z}{\sqrt{\xi}}\right)=-\frac{i}{6} \frac{z}{\sqrt{\xi}}{ }_{2} F_{1}\left[\begin{array}{rr}
2-\alpha & 1 \\
4 & ;-i \frac{z}{\sqrt{\xi}}
\end{array}\right] .
$$

For $|z|<\sqrt{\xi}$, the definition of ${ }_{2} F_{1}$ as a series yields the alternative expression:

$$
\varphi\left(\frac{z}{\sqrt{\xi}}\right)=\sum_{n=1}^{+\infty} \frac{(2-\alpha)_{n-1}}{(2+n) !}\left(-i \frac{z}{\sqrt{\xi}}\right)^{n}
$$

By definition of the sequence $\left(B_{n, k}, 1 \leq n, 1 \leq k \leq n\right)$, we have, still for $|z|<\sqrt{\xi}$ :

$$
e^{z^{2} \alpha(\alpha-1) \varphi\left(\frac{z}{\sqrt{\xi}}\right)}=1+\sum_{n=1}^{+\infty} \frac{1}{n !}\left(\frac{-i z}{\sqrt{\xi}}\right)^{n} \sum_{k=1}^{n}\left(z^{2} \alpha(\alpha-1)\right)^{k} B_{n, k}
$$

which may be read as an asymptotic expansion in $\xi$. It remains now to plug this expansion in (A.3) and integrate term by term to obtain the announced result. However, some care is needed as the convergence of the expansion is not uniform in $z$, hence we cannot apply directly [2, Theorem 1.7.5] for instance. Let $N \in \mathbb{N}$. Using the integral definition of the Gamma function, we have

$$
\begin{aligned}
& \left|\xi^{\frac{1}{2}-\frac{1}{1+\alpha}} e^{\frac{\alpha}{\alpha+1} \xi} \Phi_{\alpha}\left(\xi^{\frac{\alpha}{\alpha+1}}\right)-\frac{1}{\sqrt{2 \pi \alpha}} \sum_{p=0}^{N-1}(-1)^{p} c_{p}^{(\alpha)} \xi^{-p}\right| \\
& =\frac{1}{2 \pi}\left|\int_{-\infty}^{+\infty} e^{-\alpha \frac{z^{2}}{2}}\left(e^{z^{2} \alpha(\alpha-1) \varphi\left(\frac{z}{\sqrt{\xi}}\right)}-1-\sum_{p=1}^{N-1} \frac{(-1)^{p}}{(2 p) !}\left(\frac{z}{\sqrt{\xi}}\right)^{2 p} \sum_{k=1}^{2 p}\left(z^{2} \alpha(\alpha-1)\right)^{k} B_{2 p, k}\right) d z\right|
\end{aligned}
$$

and we need to prove that this last quantity is smaller than $K \xi^{-N}$ for some constant $K$ independent from $\xi$. We decompose the integral according as $|z|<r \sqrt{\xi}$ or $|z| \geq r \sqrt{\xi}$ where $0<r<1$ is fixed. On the one hand, when $|z|<r \sqrt{\xi}$, we use the expansion (A.4), in which we separate the even and the odd terms. For the odd terms, we obtain:

$$
\left|\int_{-r \sqrt{\xi}}^{r \sqrt{\xi}} e^{-\alpha \frac{z^{2}}{2}} \sum_{p=0}^{+\infty} \frac{(-1)^{2 p+1}}{(2 p+1) !}\left(\frac{-i z}{\sqrt{\xi}}\right)^{2 p+1} \sum_{k=1}^{2 p+1}\left(z^{2} \alpha(\alpha-1)\right)^{k} B_{2 p+1, k} d z\right|
$$

To prove that this quantity is null, we need to check that one may exchange the sum and the integral. Using Lemma A.1, we have:

$$
\begin{aligned}
& \int_{-r \sqrt{\xi}}^{r \sqrt{\xi}} e^{-\alpha \frac{z^{2}}{2}} \sum_{p=0}^{+\infty} \frac{1}{(2 p+1) !}\left(\frac{|z|}{\sqrt{\xi}}\right)^{2 p+1} \sum_{k=1}^{2 p+1}\left(z^{2} \alpha(\alpha-1)\right)^{k} B_{2 p+1, k} d z \\
& \quad \leq \int_{-r \sqrt{\xi}}^{r \sqrt{\xi}} e^{-\alpha \frac{z^{2}}{2}} \sum_{p=0}^{+\infty} r^{2 p+1} \sum_{k=1}^{2 p+1}\left(z^{2} \alpha(\alpha-1)\right)^{k} \frac{(1 / 4)^{k}}{k !} d z \\
& \quad \leq \int_{-r \sqrt{\xi}}^{r \sqrt{\xi}} e^{-\alpha \frac{z^{2}}{2}} \sum_{p=0}^{+\infty} r^{2 p+1} e^{\frac{z^{2} \alpha(\alpha-1)}{4}} d z \\
& \quad \leq \frac{2 r}{1-r^{2}} \int_{-\infty}^{+\infty} e^{-\frac{z^{2}}{4} \alpha(3-\alpha)} d z<+\infty
\end{aligned}
$$

since $\alpha \in(1,2)$. As a consequence, we may apply Fubini's theorem, which shows that (A.5) equals 0 . We now look at the even terms. Using again Lemma A.1, we obtain 
similarly:

$$
\begin{aligned}
& \left|\int_{-r \sqrt{\xi}}^{r \sqrt{\xi}} e^{-\alpha \frac{z^{2}}{2}} \sum_{p=N}^{+\infty} \frac{(-1)^{p}}{(2 p) !}\left(\frac{z}{\sqrt{\xi}}\right)^{2 p} \sum_{k=1}^{2 p}\left(z^{2} \alpha(\alpha-1)\right)^{k} B_{2 p, k} d z\right| \\
& \quad \leq \int_{-r \sqrt{\xi}}^{r \sqrt{\xi}} e^{-\alpha \frac{z^{2}}{2}} \sum_{p=0}^{+\infty} \frac{1}{(2 p+2 N) !}\left(\frac{z}{\sqrt{\xi}}\right)^{2 p+2 N} \sum_{k=1}^{2 p+2 N}\left(z^{2} \alpha(\alpha-1)\right)^{k} B_{2 p+2 N, k} d z \\
& \quad \leq \frac{1}{\xi^{N}} \int_{-r \sqrt{\xi}}^{r \sqrt{\xi}} e^{-\alpha \frac{z^{2}}{2}} \sum_{p=0}^{+\infty} z^{2 N} r^{2 p} \sum_{k=1}^{2 p+2 N}\left(z^{2} \alpha(\alpha-1)\right)^{k} \frac{(1 / 4)^{k}}{k !} d z \\
& \quad \leq \frac{1}{\xi^{N}} \sum_{p=0}^{+\infty} r^{2 p} \int_{-r \sqrt{\xi}}^{r \sqrt{\xi}} z^{2 N} e^{-\alpha \frac{z^{2}}{2}} e^{\frac{z^{2} \alpha(\alpha-1)}{4}} d z \\
& \quad \leq \frac{1}{\xi^{N}} \frac{1}{1-r^{2}} \int_{-\infty}^{+\infty} z^{2 N} e^{-\frac{z^{2}}{4} \alpha(3-\alpha)} d z
\end{aligned}
$$

where the last integral is finite and does not depend on $\xi$. On the other hand, when $|z| \geq r \sqrt{\xi}$, we apply the triangular inequality and first study:

$$
\left|\int_{|z| \geq r \sqrt{\xi}} e^{-\alpha \frac{z^{2}}{2}} e^{z^{2} \alpha(\alpha-1) \varphi\left(\frac{z}{\sqrt{\xi}}\right)} d z\right| \leq \frac{1}{r^{2 N} \xi^{N}} \int_{-\infty}^{+\infty} z^{2 N} e^{-\alpha \frac{z^{2}}{2}}\left|e^{z^{2} \alpha(\alpha-1) \varphi\left(\frac{z}{\sqrt{\xi}}\right)}\right| d z .
$$

To check that this last integral may be bounded by a constant independent of $\xi$, we shall simply prove that $\Re(\varphi(u)) \leq 0$ for any $u \in \mathbb{R}$. Indeed, observe that

$$
\varphi(u)=-\frac{i u}{2} \int_{0}^{1}(1-t)^{2}(1+i t u)^{\alpha-2} d t=-\frac{i u}{2} \int_{0}^{1} \frac{(1-t)^{2}}{\left(1+t^{2} u^{2}\right)^{\frac{2-\alpha}{2}}} e^{i \theta(2-\alpha)} d t
$$

where $\theta=\theta(t, u) \in\left[-\frac{\pi}{2}, \frac{\pi}{2}\right]$ is defined by

$$
\cos (\theta)=\frac{1}{\sqrt{1+t^{2} u^{2}}} \quad \text { and } \quad \sin (\theta)=\frac{-t u}{\sqrt{1+t^{2} u^{2}}} .
$$

In particular, the sign of $u$ is the opposite of the sign of $\theta$. Since $0<2-\alpha<1$, we deduce that the $\operatorname{sign}$ of $\sin (\theta(2-\alpha))$ is also the opposite of the sign of $u$, and thus

$$
\Re(\varphi(u))=\frac{u}{2} \int_{0}^{1} \frac{(1-t)^{2}}{\left(t^{2} u^{2}+1\right)^{\frac{2-\alpha}{2}}} \sin (\theta(2-\alpha)) d t \leq 0 .
$$

Finally, the remaining term being polynomial, we have assuming that $\xi>1$,

$$
\begin{aligned}
& \int_{|z| \geq r \sqrt{\xi}} e^{-\alpha \frac{z^{2}}{2}}\left|1+\sum_{p=1}^{N-1} \frac{(-1)^{p}}{(2 p) !}\left(\frac{z}{\sqrt{\xi}}\right)^{2 p} \sum_{k=1}^{2 p}\left(z^{2} \alpha(\alpha-1)\right)^{k} B_{2 p, k}\right| d z \\
& \leq \frac{1}{r^{2 N} \xi^{N}} \int_{-\infty}^{+\infty} z^{2 N} e^{-\alpha \frac{z^{2}}{2}}\left(1+\sum_{p=1}^{N-1} \frac{1}{(2 p) !}\left(\frac{z}{\sqrt{\xi}}\right)^{2 p} \sum_{k=1}^{2 p}\left(z^{2} \alpha(\alpha-1)\right)^{k} B_{2 p, k}\right) d z \\
& \leq \frac{1}{r^{2 N} \xi^{N}} \int_{-\infty}^{+\infty} z^{2 N} e^{-\alpha \frac{z^{2}}{2}}\left(1+\sum_{p=1}^{N-1} \frac{1}{(2 p) !} z^{2 p} \sum_{k=1}^{2 p}\left(z^{2} \alpha(\alpha-1)\right)^{k} B_{2 p, k}\right) d z
\end{aligned}
$$

and this last integral is also finite and independent from $\xi$. This ends the proof for $\Phi_{\alpha}$. 
We now study the asymptotic expansion of $\Phi_{\alpha}^{\prime}$. Since $\alpha<2$, we may differentiate under the integral in (A.1) to obtain:

$$
\begin{aligned}
\Phi_{\alpha}^{\prime}(x)=\int_{0}^{+\infty} & e^{-\sin \left(\frac{\pi \alpha}{2}\right) \frac{z^{1+\alpha}}{1+\alpha}} z \sin \left(\cos \left(\frac{\pi \alpha}{2}\right) \frac{z^{1+\alpha}}{1+\alpha}-z x\right) d z \\
& =\frac{1}{2 \pi i} \int_{-\infty}^{+\infty} z e^{i e^{i \frac{\pi \alpha}{2}} \frac{z^{1+\alpha}}{1+\alpha}-i z x} d z=\frac{1}{2 \pi i} x^{\frac{2}{\alpha}} \int_{-\infty}^{+\infty} z e^{i x^{1+\frac{1}{\alpha}}\left(e^{i \frac{\pi \alpha}{2}} \frac{z^{1+\alpha}}{1+\alpha}-z\right)} d z
\end{aligned}
$$

Using similar computations to those for $\Phi_{\alpha}$, we deduce that

$$
\Phi_{\alpha}^{\prime}(x) \underset{x \rightarrow+\infty}{\sim} \frac{1}{\sqrt{2 \pi \alpha}} x^{\frac{3-\alpha}{2 \alpha}} e^{-\frac{\alpha}{\alpha+1} x^{1+1 / \alpha}} \sum_{p=0}^{+\infty}(-1)^{p+1} d_{p}^{(\alpha)} x^{-p(1+1 / \alpha)}
$$

for some coefficients $\left(d_{p}^{(\alpha)}\right)$. The formula for these coefficients follows then by integrating the asymptotic expansion of $\left(x^{\frac{\alpha-1}{2 \alpha}} e^{\frac{\alpha}{\alpha+1} x^{1+1 / \alpha}} \Phi_{\alpha}(x)\right)^{\prime}$ and identifying the successive powers.

\section{A.3 Asymptotics of the sequence $\left(c_{p}^{(\alpha)}, p \geq 0\right)$}

Corollary A.3. There exist two constants $0<\kappa_{1}<\kappa_{2}<+\infty$ such that as $p \rightarrow+\infty$ :

$$
\kappa_{1} p \leq\left(c_{p}^{(\alpha)}\right)^{\frac{1}{p}} \leq \kappa_{2} p
$$

Proof. To get the lower bound, we simply observe that $B_{2 p, 2 p}=\left(\frac{1}{6}\right)^{2 p}$ and keep only the last term in the sum defining $c_{p}^{(\alpha)}$ :

$$
c_{p}^{(\alpha)} \geq \frac{1}{(2 p) ! \sqrt{\pi}}\left(\frac{2}{\alpha}\right)^{p}\left(\frac{1}{6}\right)^{2 p} \Gamma\left(3 p+\frac{1}{2}\right)(2(\alpha-1))^{2 p} .
$$

The result then follows from Stirling's asymptotics. To get the upper bound, we first use Lemma A.1 to write

$$
c_{p}^{(\alpha)} \leq \frac{1}{\sqrt{\pi}}\left(\frac{2}{\alpha}\right)^{p} \sum_{k=1}^{2 p} \frac{1}{k !} \Gamma\left(p+k+\frac{1}{2}\right)\left(\frac{\alpha-1}{2}\right)^{k} .
$$

Then, using the recurrence relation of the Gamma function, we obtain

$$
\begin{aligned}
\frac{\Gamma\left(p+k+\frac{1}{2}\right)}{k !} & =\left(k+\frac{1}{2}\right)\left(k+\frac{3}{2}\right) \ldots\left(k+\frac{1}{2}+p-1\right) \frac{\Gamma\left(k+\frac{1}{2}\right)}{\Gamma(k+1)} \\
& \leq\left(2 p+\frac{1}{2}\right)\left(2 p+\frac{3}{2}\right) \ldots\left(2 p+\frac{1}{2}+p-1\right) \\
& \leq(3 p)^{p} .
\end{aligned}
$$

As a consequence,

$$
\sum_{k=1}^{2 p}\left(\frac{\alpha-1}{2}\right)^{k} \frac{\Gamma\left(p+k+\frac{1}{2}\right)}{k !} \leq \frac{\alpha-1}{3-\alpha}(3 p)^{p}
$$

which ends the proof.

\section{References}

[1] E. Barkai, E. Aghion, D.A. Kessler. From the area under the Bessel excursion to anomalous diffusion of cold atoms. Phys. Rev. X 4 (2), 021036, 2014. 
The area under a spectrally positive stable excursion

[2] N. Bleistein and R. Handelsman. Asymptotic Expansions of Integrals. Second edition. Dover Publications, Inc., New York, 1986. MR-0863284

[3] A. N. Borodin and P. Salminen. Handbook of Brownian motion-facts and formulae. Probability and its Applications. Birkhäuser Verlag, Basel, second edition, 2002. MR-1912205

[4] L. Chaumont. Excursion normalisée, méandre et pont pour les processus de Lévy stables. (French) [Normalized excursion, meander and bridge for stable Lévy processes] Bull. Sci. Math. 121, no. 5, 377-403, 1997. MR-1465814

[5] L. Comtet. Advanced Combinatorics. The Art of Finite and Infinite Expansions. Revised and enlarged edition. D. Reidel Publishing Co., Dordrecht, 1974. MR-0460128

[6] P. Flajolet and G. Louchard. Analytic variations on the Airy distribution. Algorithmica 31, 361-377, 2001. MR-1855255

[7] R. Gorenflo, Y. Luchko, and F. Mainardi. Analytical properties and applications of the Wright function. Fract. Calc. Appl. Anal. 2, no. 4, 383-414, 1999. MR-1752379

[8] S. Janson. Brownian excursion area, Wright's constants in graph enumeration, and other Brownian areas. Probab. Surv. 4, 80-145, 2007. MR-2318402

[9] S. Janson. Moments of gamma type and the Brownian supremum process area. Probab. Surv. 7, 1-52, 2010. MR-2645216

[10] S. Janson and G. Louchard. Tail estimates for the Brownian excursion area and other Brownian areas. Electron. J. Probab. 12, no. 58, 1600-1632, 2007. MR-2365879

[11] Y. Kasahara. Tauberian theorems of exponential type. J. Math. Kyoto Univ. 18, no. 2, 209-219, 1978. MR-0501841

[12] D. A. Kessler, S. Medalion and E. Barkai. The distribution of the area under a Bessel excursion and its moments. J. Stat. Phys. 156, 686-706, 2014. MR-3226838

[13] A. Lachal. Sur le premier instant de passage de l'intégrale du mouvement brownien. (French) [First hitting time of integrated Brownian motion]. Ann. Inst. H. Poincaré Probab. Statist. 27, no. 3, 385-405, 1991. MR-1131839

[14] M. Lefebvre. First passage densities of a two-dimensional process. S.I.A.M. J. Appl. Math. 49, 1514-1523, 1989. MR-1015076

[15] J. Letemplier and T. Simon. The area of a spectrally positive stable process stopped at zero. Probab. Math. Statist. 38, no. 1, 27-37, 2018. MR-3813904

[16] G. Louchard. Kac's formula, Lévy's local time and Brownian excursion. J. Appl. Probab. 21, no. 3, 479-499, 1984. MR-0752014

[17] G. Louchard. The Brownian excursion area: a numerical analysis. Comput. Math. Appl. 10, no. 6, 413-417, 1984. MR-0783514

[18] F. Mainardi, A. Mura and G. Pagnini. The $M$-Wright function in time-fractional diffusion processes: a tutorial survey. Int. J. Differ. Equ. Art. ID 104505, 29 pp., 2010. MR-2592742

[19] S. N. Majumdar and A. Comtet. Airy distribution function: from the area under a Brownian excursion to the maximal height of fluctuating interfaces. J. Stat. Phys. 119, no. 3-4, 777-826, 2005. MR-2151223

[20] D. Monrad and M. L. Silverstein. Stable processes: sample function growth at a local minimum. Z. Wahrsch. Verw. Gebiete 49, no. 2, 177-210, 1979. MR-0543993

[21] M. Perman and J. A. Wellner. On the distribution of Brownian areas. Ann. Appl. Probab. 6, no. 4, 1091-1111, 1996. MR-1422979

[22] G. Samorodnitsky and M. S. Taqqu. Stable Non-Gaussian Random Processes. Chapman \& Hall, New-York, 1994 MR-1280932

[23] K. Sato. Lévy Processes and Infinitely Divisible Distributions. Cambridge University Press, Cambridge, 1999. MR-1739520

[24] L. Takács. A Bernoulli excursion and its various applications. Adv. Appl. Probab. 23, no. 3, 557-585, 1991. MR-1122875

[25] L. Takács. Limit distributions for the Bernoulli meander. J. Appl. Probab. 32, no. 2, 375-395, 1995. MR-1334893 
The area under a spectrally positive stable excursion

[26] E. M. Wright. The generalized Bessel function of order greater than one. Quart. J. Math., Oxford ser. 11, 36-48 1940. MR-0003875

Acknowledgments. We wish to thank the referees for pointing out some gaps in an earlier version of this manuscript. 


\section{Electronic Journal of Probability Electronic Communications in Probability}

\section{Advantages of publishing in EJP-ECP}

- Very high standards

- Free for authors, free for readers

- Quick publication (no backlog)

- Secure publication $\left(\mathrm{LOCKSS}^{1}\right)$

- Easy interface (EJMS²)

\section{Economical model of EJP-ECP}

- Non profit, sponsored by $\mathrm{IMS}^{3}, \mathrm{BS}^{4}$, ProjectEuclid ${ }^{5}$

- Purely electronic

\section{Help keep the journal free and vigorous}

- Donate to the IMS open access fund ${ }^{6}$ (click here to donate!)

- Submit your best articles to EJP-ECP

- Choose EJP-ECP over for-profit journals

\footnotetext{
${ }^{1}$ LOCKSS: Lots of Copies Keep Stuff Safe http://www. lockss.org/

${ }^{2}$ EJMS: Electronic Journal Management System http://www.vtex.lt/en/ejms.html

${ }^{3}$ IMS: Institute of Mathematical Statistics http://www.imstat.org/

${ }^{4}$ BS: Bernoulli Society http://www. bernoulli-society.org/

${ }^{5}$ Project Euclid: https://projecteuclid.org/

${ }^{6}$ IMS Open Access Fund: http://www.imstat.org/publications/open.htm
} 